\title{
Properties of stellar clusters around high-mass young stars $\mathbf{s}^{\star} \star \star$
}

\author{
F. Faustini ${ }^{1}$, S. Molinari ${ }^{1}$, L. Testi ${ }^{2,3}$, and J. Brand ${ }^{4}$ \\ 1 Istituto di Fisica dello Spazio Interplanetario - INAF, via Fosso del Cavaliere 100, 00133 Rome, Italy \\ e-mail: fabiana.faustini@ifsi-roma.inaf.it \\ 2 European Southern Observatory, Karl Schwarzschild str. 2, Garching bei Muenchen, Germany \\ e-mail: 1testi@eso.org \\ 3 Osservatorio Astronomico di Arcetri - INAF, via Enrico Fermi 5, 50125 Firenze, Italy \\ ${ }^{4}$ Istituto di Radioastronomia - INAF, via Gobetti 101, Bologna, Italy \\ e-mail: brand@ira.inaf.it
}

Received 26 November 2007 / Accepted 1 April 2009

ABSTRACT

\begin{abstract}
Context. Twenty-six high-luminosity IRAS sources believed to be collection of stars in the early phases of high-mass star formation have been observed in the near-IR $\left(J, H, K_{\mathrm{s}}\right)$ to characterize the clustering properties of their young stellar population and compare them with those of more evolved objects (e.g., Herbig Ae/Be stars) of comparable mass. All the observed sources possess strong continuum and/or line emission in the millimeter, being therefore associated with gas and dust envelopes. Nine sources have far-IR colors characteristic of UCHII regions, while the other 17 are probably experiencing an evolutionary phase that precedes the hot-cores, as suggested by a variety of evidence collected in the past decade.

Aims. We attempt to gain insight into the initial conditions of star formation in these clusters (initial mass function [IMF], star formation history $[\mathrm{SFH}])$, and to determine mean cluster ages.

Methods. For each cluster, we complete aperture photometry. We derive stellar density profiles, color-color and color-magnitude diagrams, and color (HKCF) and luminosity (KLF) functions. These two functions are compared with simulated KLFs and HKCFs from a model that generates populations of synthetic clusters starting from assumptions about the IMF, SFH, and Pre-MS evolution, and using the average properties of the observed clusters as boundary conditions (bolometric luminosity, dust distribution, infrared excess, extinction).

Results. Twenty-two sources show evidence of clustering with a stellar richness indicator that varies from a few up to several tens of objects, and a median cluster radius of $0.7 \mathrm{pc}$. A considerable number of cluster members present an infrared excess characteristic of young pre-main-sequence objects. For a subset of 9 detected clusters, we could perform a statistically significant comparison of the observed KLFs with those resulting from synthetic cluster models; for these clusters, we find that the median stellar age ranges between $2.5 \times 10^{5}$ and $5 \times 10^{6}$ years, with evidence of an age spread of the same entity within each cluster. We also find evidence that older clusters tend to be smaller in size, in agreement with our clusters being on average larger than those around relatively older Herbig Ae/Be stars. Our models allow us to explore the relationship between the mass of the most massive star in the cluster and both the cluster richness and the total stellar mass. Although these relationships are predicted by several classes of cluster formation models, their detailed analysis suggests that the properties of our modeled clusters may not be consistent with them resulting from random sampling of the IMF.

Conclusions. Our results are consistent with star formation having occurred continuously over a period of time longer than the typical crossing time.
\end{abstract}

Key words. stars: formation - stars: imaging - stars: luminosity function, mass function - stars: pre-main sequence - infrared: stars

\section{Introduction}

There have been considerable efforts to understand how stars form from both a theoretical and an observational point of view. We have reached a good understanding of how isolated lowmass stars form (Klein et al. 2006). The widely accepted scenario is that low-mass stars form by the gravitational collapse of a prestellar core followed at later stages by disk accretion.

Extending this theory to high-mass stars is not trivial. Highmass (proto-)stars reach the zero age main sequence while still accreting. When the central protostar reaches a mass of about $10 M_{\odot}$ hydrogen fusion ignites in the core and the star's

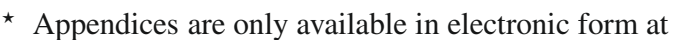
http://www . aanda.org

$\star \star$ Based on observations obtained at the Palomar Observatory and at the ESO La Silla Observatory (Chile), programme 65.I-0310(A).
}

radiation pressure and wind should prevent further accretion. This is obviously a paradox given that yet more massive stars do form. Several theories have been put forward to solve this dilemma (Zinnecker \& Yorke 2007), such as accretion rates of up to three orders of magnitude higher than in the case of lowmass stars (Cesaroni 2005), and non-spherical accretion geometries (Nakano 1989; Yorke 2002; Keto 2003), or coalescence in dense (proto-)stellar clusters (Bonnell et al. 1998).

All of these theories have predictions that can, in principle, be tested observationally. Significant effort has been made to detect massive accretion disks (Cesaroni et al. 2006), powerful outflows (Beuther et al. 2002; Cesaroni et al. 2005), and dense protostellar clusters (Testi et al. 1999; de Wit et al. 2005), all of which are predicted by one or other formation theory. None of these efforts have provided conclusive arguments in favour or against any of the theories. 
In this paper we explore the properties of embedded clusters associated with high-mass protostellar candidates. Our sample was selected from a larger sample of candidate high-mass protostars selected and analyzed by Molinari et al. (1996, 1998, 2000, 2002) and Brand et al. (2001). In Sect. 2, we present the observations and data analysis (source extraction, photometry), and in Sect. 3, we discuss data elaboration and interpretation. In Sect. 4, we present our Synthetic Cluster Generation model and the method of comparison between synthetic and observed clusters and the results of using this technique. Finally, in Sect. 5 we compare our objects with more evolved ones and present our conclusions.

\section{Observations and data analysis}

Program fields are listed in Table 1 and were imaged in $J, H$, and $K_{\mathrm{s}}$ bands. A total of 15 fields were observed in three nights in November 1998 at the Palomar 60-inch telescope equipped

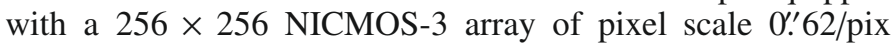
and total FOV 2! $6 \times 2$. 6 . The remaining 11 fields were observed in 3 nights in August 2000 at the ESO-NTT using the $1024 \times 1024$ SOFI camera with a pixel scale of $0.29 /$ pix and a total FOV of $4.9 \times 44^{\prime} .9$. Standard dithering techniques were used to minimize the impact of bad pixels and optimize flat-fielding, allowing us to achieve for each field a total of $5 \mathrm{~min}$ integration time per band (in the central portion of the observed field) within an area of 3.5 $\times 3.5$ of Palomar observations, and $20 \mathrm{~min}(10 \mathrm{~min}$ for the band $K_{\mathrm{s}}$ at NTT of a total covered area of $6.5 \times 6.5$. Suitable calibration sources from the list of Hunt et al. (1988) were observed regularly during the observations to track atmospheric variations for different airmasses. Standard stars and target fields were observed at airmasses no greater than 1.7 at NTT, and 1.3 at Palomar; we determined average zero-point magnitudes for each night and used them to calibrate our photometry. For each field, the images in the three bands were registered and astrometric solutions were determined using a few bright optically visible sources.

The $K_{\mathrm{s}}$ images for all observed fields, with superimposed submillimeter continuum emission distribution when available (Molinari et al. 2008a) are presented in Appendix A, and are also available online ${ }^{1}$.

\subsection{Point source extraction and photometry}

The extraction and photometry of point sources for all images were completed using the IRAF package. The rms of the background signal and the $F W H M$ of point sources were measured throughout the images to characterize the image noise and PSF properties; these parameters were fed to the DAOFIND task for source extraction, where a detection threshold of $3 \sigma$ was used for all images. Sources with saturated pixels were excluded from the analysis; the linearity of the system response was checked a posteriori comparing, both for the Palomar and the NTT data, the magnitudes obtained to those from 2MASS using a few stars with magnitudes reaching up to the maximum values found in our photometry files; the relations between the 2MASS magnitudes and ours in the three bands were found to be linear over the entire magnitude range of the detected sources. There were clearly brighter objects in the various fields, but their peaks were already flagged as saturated and excluded from the detection process.

\footnotetext{
1 At K-images/.
}

Table 1. Description of observations.

\begin{tabular}{lcccc}
\hline \hline $\begin{array}{l}\text { Source } \\
\mathrm{Mol}^{a}\end{array}$ & IRAS Name & $\alpha(\mathrm{J} 2000)$ & $\delta(\mathrm{J} 2000)$ & Tel. \\
\hline 3 & $00420+5530$ & $00: 44: 57.6$ & $+55: 46: 52$ & $\mathrm{Pal}$ \\
8 & $05137+3919$ & $05: 17: 13.3$ & $+39: 22: 14$ & $\mathrm{Pal}$ \\
9 & $05168+3634$ & $05: 20: 16.2$ & $+36: 37: 21$ & $\mathrm{Pal}$ \\
11 & $05345+3157$ & $05: 37: 47.8$ & $+31: 59: 24$ & $\mathrm{Pal}$ \\
12 & $05373+2349$ & $05: 40: 24.4$ & $+23: 50: 54$ & $\mathrm{NTT}$ \\
15 & $06056+2131$ & $06: 08: 41.0$ & $+21: 31: 01$ & $\mathrm{Pal}$ \\
28 & $06584-0852$ & $07: 00: 51.0$ & $-08: 56: 29$ & $\mathrm{Pal}$ \\
30 & $17450-1742$ & $17: 48: 09.3$ & $-27: 43: 21$ & $\mathrm{NTT}$ \\
38 & $18024-2119$ & $18: 06: 18.0$ & $-21: 42: 00$ & $\mathrm{NTT}$ \\
45 & $18144-1723$ & $18: 17: 24.2$ & $-17: 22: 13$ & NTT \\
50 & $18162-1612$ & $18: 19: 07.5$ & $-16: 11: 21$ & NTT \\
59 & $18278-1009$ & $18: 30: 35.2$ & $-10: 07: 12$ & Pal \\
75 & $18511+0146$ & $18: 53: 38.1$ & $+01: 50: 27$ & Pal \\
82 & $18565+0349$ & $18: 59: 03.4$ & $+03: 53: 22$ & NTT \\
84 & $18567+0700$ & $18: 59: 13.6$ & $+07: 04: 47$ & NTT \\
98 & $19092+0841$ & $19: 11: 37.4$ & $+08: 46: 30.0$ & NTT \\
99 & $19094+0944$ & $19: 11: 52.0$ & $+09: 49: 46$ & Pal \\
103 & $19213+1723$ & $19: 23: 37.0$ & $+17: 28: 59$ & NTT \\
109 & $19374+2352$ & $19: 39: 33.2$ & $+23: 59: 55$ & NTT \\
110 & $19388+2357$ & $19: 40: 59.4$ & $+24: 04: 39$ & NTT \\
136 & $21307+5049$ & $21: 32: 31.5$ & $+51: 02: 22.0$ & Pal \\
139 & $21519+5613$ & $21: 53: 38.8$ & $+56: 27: 53.0$ & Pal \\
143 & $22172+5549$ & $22: 19: 09.0$ & $+56: 04: 45.0$ & Pal \\
148 & $22305+5803$ & $22: 32: 24.3$ & $+58: 18: 58.2$ & Pal \\
151 & $22506+5944$ & $22: 52: 38.6$ & $+60: 00: 56.0$ & Pal \\
160 & $23385+6053$ & $23: 40: 53.3$ & $+61: 10: 19.1$ & Pal \\
\hline
\end{tabular}

${ }^{a}$ Source running number from Molinari et al. (1996).

${ }^{b}$ Imaged only in $K_{\mathrm{s}}$.

The photometry of sources is difficult to determine in very dense stellar fields such as the inner Galactic plane, where all our target fields are located and the crowding is such that more than one source can enter either any plausible aperture chosen or any annulus used for background estimation. This problem is of course more extreme in the clustered environments close to detected sites of massive star formation (see Sect. 3.1 below).

The first alternative approach that we tried to follow was PSF-fitting photometry that should be less affected by these problems. We chose a subsample of test fields with different levels of stellar crowding. In this procedure, an important aspect was the modeling of the PSF. To test this, we completed several trials selecting a variable number of point-like sources (from 3 to 30) of different brightness levels and different positions in the field. We found that the resulting PSF model was not particularly sensitive to the choice of numbers and/or brightnesses of the stars. However the results, were quite dependent on the mean stellar density of the field. The photometry was carried out using the ALLSTAR task, which was particularly suited to crowded fields. However, we also tested the other two tasks (PEAK and NSTAR) and obtained comparable results for most of the sources. We note, however, that in the most crowded areas in particular, the subtraction of the PSF-fitted sources from the image introduced two spurious effects: an unacceptably high level of residuals with brightness levels well above the detection threshold used and a significant number of negative holes, indicating that the PSF-fit included some background in the source flux estimation and therefore overestimated its value. Both effects are caused by both the limited accuracy of the PSF model that can be obtained for very crowded fields, where faint neighboring stars can enter the area where the PSF model is estimated, and the presence of a significant and variable background, which 
is quite common and expected for the Galactic plane. A similar conclusion was reached by Hillenbrand \& Carpenter (2000) in their study of the inner Orion Nebula Cluster.

The second approach that we followed was standard aperture photometry. The choice of radii for both the aperture and the background annuli was of course extremely important. The optimum aperture should be neither too large to include nearby sources nor too small to truncate significantly the PSF and underestimate the flux significantly. We completed several attempts for one of the most crowded fields (Mol30, observed at NTT) with three different aperture radii equal to the PSF FWHM (typically $0 . ' 7$ at NTT and 1.' 4 at Palomar in $K_{\mathrm{s}}$ ), and twice and thrice this value. For each photometry run, we analyzed the source flux distribution and, as expected, the median flux was found to increase with increasing aperture radius. Increasing the aperture from one to two PSF FWHMs increased the median source flux by an amount compatible with the inclusion of the first ring of an Airy diffraction pattern. In contrast, when the aperture radius was increased to a factor of three higher than the PSF $F W H M$, the flux increase was far higher than could be attributed to the additional fraction of the Airy profile entering the aperture, and must therefore have been caused by the inclusion of nearby sources. We adopted an aperture radius equal to the PSF $F W H M$ to minimize neighbor contamination, and then applied an aperture correction factor to the fraction of the PSF removed by the aperture; this was estimated by multi-aperture photometry (starting from a size of $1 F W H M$ ) on relatively isolated stars in the target fields.

Given the crowding of our fields, a further effect to be corrected for is the possible contamination by the tails of the brightness profiles of neighbouring stars. To quantify this contamination, we created a grid of simulations with two symmetric Gaussians with a wide variety of peak contrasts and different reciprocal distances. We computed the fraction of the Gaussian profile of the neighbouring source within the photometry aperture centered on the main source, and hence generated a matrix of photometry corrections for different source distances and peak contrasts. We then processed the magnitude file produced by the aperture photometry task and for each source we applied a magnitude correction depending on the presence, distance, and contrast ratios with respect to other neighbouring stars.

In spite of the various issues discussed above, the photometric data obtained with the two methods were in good agreement with each other, apart from at faint magnitudes. For these faint objects, we consistently found that the PSF photometry tends to produce brighter magnitudes (and hence stronger sources) than the aperture photometry; this effect can be easily understood from our finding (see above) that the subtraction of PSF-fitted sources always leaves negative holes in the residual image, and this effect is far more important for faint stars. We thus decided to adopt the magnitudes determined from aperture photometry.

For each target field, we estimated the limiting magnitude (LM) using artificial star experiments. The fields were populated using the IRAF task ADDSTAR with 400 fake stars with magnitudes distributed in bins of 0.25 mag between values of 15 and 21 ; the percentage of recovered stars as a function of magnitude provides an estimate of the completeness level of our photometry. The star recovery percentage was not found to decrease monotonically with increasing magnitude because fake stars can also be placed very close to bright real stars and then go undetected by the finding algorithm. However, we find that the limit of $85-90 \%$ recovery fraction is reached on average at around $J=18.7, H=17.7$, and $K_{\mathrm{s}}=17.4$ for NTT images, and $J=18.0, H=17.3$, and $K_{\mathrm{s}}=16.6$ for Palomar images. We found that the typical photometric uncertainty is below $0.1 \mathrm{mag}$ close to the limiting magnitude.

To verify the integrity of our photometry, we compared our magnitudes with those extracted from 2MASS point source cata$\log$ for all the fields in our sample. Considering the differences in spatial resolutions between 2MASS and the telescopes used for our observations, this comparison was limited to 2MASS pointlike sources associated with a single source in the Palomar or NTT images. The median differences with respect to 2MASS for the various fields are of the order of $-0.1,-0.2$ and $-0.3 \mathrm{mag}$ for $J, H$, and $K_{\mathrm{s}}$ bands, respectively. Within each field, the scatter around these median values is $\sim 0.1 \mathrm{mag}$ in all three bands, confirming the internal consistency of our photometry. Noticeable departures $(\sim 0.5 \mathrm{mag})$ of the median difference with 2MASS from the above values are observed for the field of source Mol11 (Palomar), and for sources Mol103, Mol109 and Mol110 (NTT). However, the latter sources were observed on the same night, observations for which our log registered as not good due to sky variations that were not tracked by night-averaged zero points. We emphasize again, however, that these are systematic differences with respect to $2 \mathrm{MASS}$ in this limited number of cases; the rms scatter about these median differences are $\sim 0.1$ mag in all bands and this should provide confidence that the internal consistency of the photometry in each field is preserved. We then decided to rescale our photometry to the 2MASS photometric system to remove these systematic effects. The $(J-H)$ and $(H-K)$ color differences between 2MASS and our photometry are not correlated with the magnitude, so that no magnitude-dependent color effect is introduced in this rescaling.

\section{Results}

\subsection{Cluster Identification}

The identification of a cluster results from the analysis of stellar density in the field. Since our target fields are sites of massive star formation associated with local peaks of dust column densities and hence of visual extinction, the $K_{\mathrm{s}}$ images are clearly more suited for this type of analysis.

Stellar density maps were compiled for each field by counting stars in a running boxcar of size equal to $20^{\prime \prime}$. The box size was determined empirically to enhance the statistical significance of local stellar density peaks and to maximize the ability to detect the clusters. Larger boxes tend to smear the cluster into the background stellar density field decreasing the statistical significance of the peak, which may lead to non-detection of a clearly evident cluster, particularly in the rich inner Galaxy fields (this happens, e.g., for source Mol103, see Fig. A.18 in the Appendix). Smaller boxes produce noisy density maps where the number of sources in each bin starts to be comparable to the fluctuations in the background density field caused either by intrinsic variations in the field star density or to variable extinction from diffuse foreground ISM in the Galactic Plane (where all of our sources are located). For most of our objects in the outer Galaxy, this analysis is used to locate the position of the peak stellar density, since the clusters are obvious already from visual inspection (Mol3 to Mol28, and Mol143 to Mol151, see Appendix). For the remaining fields, the density maps are used to ascertain the presence of a cluster; toward the inner Galaxy in particular, the density maps tend to show more than one peak at comparable levels. It is important to remember, however, that this is a search for stellar clusters toward regions where indications of active star formation are already available, and this information can be used. In particular, the coincidence of these 
Table 2. Results for cluster detection.

\begin{tabular}{|c|c|c|c|c|c|c|c|c|c|c|c|}
\hline \multirow{3}{*}{$\begin{array}{l}\text { Sou. } \\
\text { Mol }\end{array}$} & \multirow{3}{*}{$\begin{array}{c}d^{a} \\
\mathrm{kpc}\end{array}$} & \multirow[t]{3}{*}{$I_{\mathrm{c}}$} & \multirow[t]{3}{*}{$N_{\text {obs }}$} & \multirow{3}{*}{$\begin{array}{l}R_{\text {clu }} \\
\text { pc }\end{array}$} & \multicolumn{2}{|c|}{ Pre-MS } & \multirow{3}{*}{$\begin{array}{c}M_{\mathrm{gas}} \\
M_{\odot}\end{array}$} & \multirow{3}{*}{$\begin{array}{c}L_{\text {bol }} \\
10^{3} L_{\odot}\end{array}$} & \multirow{3}{*}{$\begin{array}{c}A_{V \text { peak }} \\
\text { mag }\end{array}$} & \multicolumn{2}{|c|}{ Cluster Center } \\
\hline & & & & & $\%$ & $\%$ & & & & $\alpha(\mathrm{J} 2000)$ & $\delta(\mathrm{J} 2000)$ \\
\hline & & & & & $C C$ & $C M$ & & & & & \\
\hline 3 & 2.17 & 78 & 78 & 1.7 & 34 & 99 & 910 & 12.4 & 18 & $00: 44: 57.4$ & $+55: 47: 20.0$ \\
\hline $8 \mathrm{~A}$ & 11.5 & 25 & 30 & 1.3 & 37 & 27 & 1650 & 57.0 & 18 & $05: 17: 13.8$ & $+39: 22: 29.7$ \\
\hline $8 \mathrm{~B}$ & 11.5 & 27 & 24 & 1.3 & 9 & 4 & 1780 & 5.5 & 8 & $05: 17: 12.0$ & $+39: 21: 51.8$ \\
\hline 9 & 6.2 & 7 & 7 & 0.6 & 16 & $-g$ & - & 24 & - & $05: 20: 16.9$ & $+36: 37: 22.0$ \\
\hline 11 & 2.1 & 51 & 48 & 0.5 & 12 & 41 & 360 & 4.6 & 35 & $05: 37: 47.7$ & $+31: 59: 24.0$ \\
\hline 12 & 1.6 & 12 & 13 & 0.3 & 0 & 30 & 72 & 1.6 & 46 & $05: 40: 24.4$ & $+23: 51: 54.8$ \\
\hline 15 & 1.5 & 64 & 61 & 0.3 & 34 & $-^{g}$ & - & 5.8 & - & 06:08:41.0 & $+21: 31: 00.0$ \\
\hline 28 & 4.5 & 75 & 75 & 1.0 & 58 & 95 & 220 & 9.1 & 4 & $07: 00: 51.5$ & $-08: 56: 18.2$ \\
\hline 30 & 0.3 & $200^{e}$ & \multicolumn{4}{|c|}{ no cluster detected ${ }^{b}$} & - & 0.14 & - & - & - \\
\hline 38 & 0.5 & -5 & \multicolumn{4}{|c|}{ no cluster detected ${ }^{c}$} & $-^{h}$ & 0.19 & 40 & - & - \\
\hline 45 & 4.3 & 28 & 27 & 0.5 & 68 & 19 & 1340 & 21.2 & 77 & $18: 17: 24.1$ & $-17: 22: 12.3$ \\
\hline 50 & 4.9 & 46 & 43 & 0.6 & 36 & 41 & 80 & 17.3 & 25 & 18:19:07.6 & $-16: 11: 21.0$ \\
\hline 59 & 5.7 & 0 & \multicolumn{4}{|c|}{ no cluster detected ${ }^{c}$} & $-^{h}$ & 11 & 29 & - & - \\
\hline 75 & 3.9 & 8 & 7 & 0.7 & 37 & 24 & 1310 & 13.3 & 38 & 18:53:38.1 & $+01: 50: 26.5$ \\
\hline 82 & 6.8 & 8 & 10 & 0.3 & 0 & 52 & 590 & 15.4 & 52 & $18: 59: 03.2$ & $+03: 53: 16.7$ \\
\hline 84 & 2.2 & 21 & 21 & 0.3 & 0 & 54 & 28 & 4.3 & 15 & $18: 59: 14.3$ & $+07: 04: 52.3$ \\
\hline 98 & 4.5 & 6 & \multicolumn{4}{|c|}{ no cluster detected ${ }^{f}$} & $-^{h}$ & 9.2 & 68 & - & - \\
\hline 99 & 6.1 & 45 & 38 & 0.9 & 49 & $-{ }^{g}$ & - & 37.3 & - & 19:11:51.4 & $+09: 49: 35.4$ \\
\hline 103 & 4.1 & 105 & 107 & 0.7 & 46 & 87 & 510 & 28.2 & 42 & $19: 23: 36.2$ & $+17: 28: 58.1$ \\
\hline 109 & 4.3 & 19 & 17 & 0.5 & $d$ & $d$ & 1030 & 26.7 & 90 & $19: 39: 33.0$ & $+24: 00: 21.3$ \\
\hline 110 & 4.3 & 23 & 20 & 0.5 & $d$ & $d$ & 400 & 14.8 & 55 & $19: 40: 58.5$ & $+24: 04: 36.3$ \\
\hline 136 & 3.6 & 21 & 19 & 0.6 & 18 & 52 & 230 & 4 & 21 & $21: 32: 31.4$ & $+51: 02: 23.1$ \\
\hline 139 & 7.3 & 25 & 24 & 1.2 & 10 & 31 & 1870 & 1.35 & 20 & $21: 53: 39.2$ & $+56: 27: 50.7$ \\
\hline 143 & 5.0 & 25 & 22 & 0.8 & 10 & 76 & 630 & 7.8 & 26 & 22:19:09.0 & $+56: 04: 58.7$ \\
\hline 148 & 5.1 & 43 & 41 & 0.9 & 43 & 64 & 22 & 7.8 & 13 & $22: 32: 23.4$ & $+58: 19: 01.3$ \\
\hline 151 & 5.4 & 15 & 14 & 0.9 & 12 & 30 & 2020 & 25 & 40 & $22: 52: 38.3$ & $+60: 00: 44.6$ \\
\hline 160 & 5.0 & 36 & 34 & 1.3 & 30 & 76 & 1830 & 16 & 32 & $23: 40: 53.1$ & $+61: 10: 21.0$ \\
\hline
\end{tabular}

${ }^{a}$ Kinetic distance using the rotation curve from Brand \& Blitz (1993).

${ }^{b}$ Stellar density analysis inconclusive due to extreme crowdedness of this field.

${ }^{c}$ Stellar density reveales no peaks close to the IRAS position or the submm peak.

${ }^{d}$ Only observed in $K_{\mathrm{s}}$.

${ }^{e}$ Detection refused due to extreme field complexity (see text).

${ }^{f}$ Detection refused because only 1 annulus in the radial density profile is above background (see text).

${ }^{g}$ No extinction estimate is available due to lack of submm information to evaluate de-reddening correction.

${ }^{h}$ Extinction estimate is available from single-pointing submillimeter data (Molinari et al. 2000) but not from maps, so that a reliable clump mass estimate is not possible.

peaks with cold dust clumps traced by intense submillimeter and millimeter emission (Beltrán et al. 2006; Molinari et al. 2008a) is critical before we can consider the density peak to be a true feature associated with the star formation region. Casual association is excluded by the high number of positive associations (see Table 2).

As further confirmation of the positive detection of a cluster we compiled radial stellar density profiles where stars were counted inside annuli of increasing internal radius and constant width and then divided by the area of the annuli (Testi et al. 1998); uncertainties were assigned assuming Poisson statistics for the number of stars in each annulus. We then assigned a positive cluster identification if the radial profile exhibited at least two annuli that had values above the background. To refine the location of the density peak, we repeated the radial density profile analysis starting from several locations within $10^{\prime \prime}$ of the peak derived from the density maps; the location that maximizes the overall statistical significance of the annuli was then assigned to the cluster center. Figure 1 shows the typical footprint of a cluster, where the stellar density is plotted as a function of distance $r$ from the start location; the density has a maximum at $r=0$ and decreases until it reaches a constant value, which is the average background/foreground stellar density.
There were two exceptions in this analysis. The first was for source Mol160. The $K_{\mathrm{s}}$-band image shows clear stellar density enhancement in a semi-circular annulus surrounding the northern side of the dense millimeter core (see Fig. A.26 in the Appendix), which appears devoid of stars. This stellar density enhancement is coincident with the emission patterns visible in the mid-IR (Molinari et al. 2008b), so is clearly a stellar population associated with the star-forming region. Since the millimeter peak is at the center of symmetry of the semi-circular stellar distribution, we consider this tobe the center of the cluster. This is only for completeness, since we cannot say whether the low density of stars at the millimeter peak is an effect of extreme visual extinction or reflects an intrinsic paucity of NIR-visible forming stars, as the proposed extreme youth of the massive YSO accreting in its depth would seem to suggest (Molinari et al. 2008b).

The second exception was for source Mol8. The stellar density analysis shows two peaks that are coincident with two distinct dust cores (see Fig. A.2); we therefore assumed the presence of two distinct clusters, rather than a subclustering feature within the same cluster. The radial density profile analysis could not be used here, so we fit elliptical Gaussians to the peaks in the density maps, allowing for an underlying constant level representing the background stellar density. The resulting cluster 


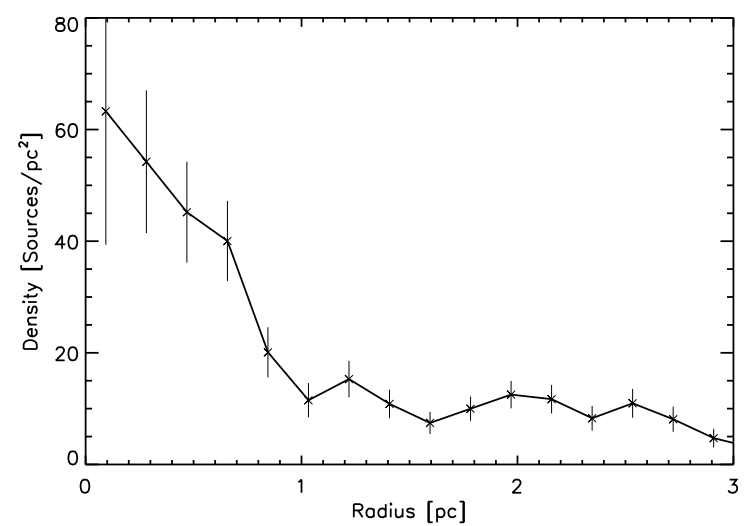

Fig. 1. Stellar density (in stars $/ \mathrm{pc}^{2}$ ), for Mol28, as a function of the radial distance (in parsecs) from cluster center. Error-bars are computed as the Poissonian fluctuations of source counts in each bin.

richness was obtained by integrating the fitted Gaussian, and the cluster radius was taken to be equal to the fitted FWHM (the fitted Gaussians were nearly circular).

Always following Testi et al. (1998), we determined the richness indicator of the cluster $I_{\mathrm{c}}$ by integrating the backgroundsubtracted density profile; the cluster radius was taken to be the radial distance from the start location where the density profile reaches a constant value. This richness indicator is a very convenient figure to use when no detailed information is available for each single star in the region and the membership of the cluster cannot be established for each single star. These values are reported in Col. 3 of Table 2 for all fields where a cluster has been clearly revealed. Column 1 gives the target name (cf. Table 1); its kinematic distance is listed in Col. 2. The parameter $N_{\text {obs }}(\mathrm{Col} .4)$ is the number of cluster members derived (see Sect. 4.1 below) from the integration of the background-subtracted $K_{\mathrm{S}}$ luminosity function (hereafter KLF, see Sect. 4.1). Also reported in Col. 8 is the mass of the hosting molecular clump; this was derived from the cold dust emission as reported in Molinari et al. (2008a, 2000), integrated over the entire spatial extent of the cluster; conversion into masses was achieved based on the optically thin assumption and by assuming $T=30 \mathrm{~K}, \beta=1.5$ (Molinari et al. 2008a), and a mass opacity $\kappa_{230 \mathrm{GHz}}=0.005 \mathrm{~cm}^{2} \mathrm{~g}^{-1}$ which corresponds to a gas/dust weight ratio of 100 (Preibisch et al. 1993). The IRAS source bolometric luminosity, Col. 9, is taken from Molinari et al. (1996, 2000, 2002, 2008a); in Col. 10 we list the $A_{V}$ at the peak cluster position estimated from submm observations (Molinari et al. 2008a, 2000). In Cols. 11 and 12 the coordinates of the centers of the identified clusters are reported. Columns 6 and 7 contain parameters that are described later in the text (see Sect. 3.2).

Following the procedure described, a cluster was detected within $1^{\prime}$ of the IRAS position for 22 out of the 26 observed fields (85\% detection rate). In two cases (Mol38 and Mol59), the stellar density map does not show a clear peak above the fluctuations of the field stellar density. For Mol98, the radial density profile only shows one annulus above the background, and therefore fails the criterion that the stellar density enhancement should be resolved significantly above the background in two annuli. In one case (Mol30), several stellar density peaks were found in proximity to the IRAS source, but the lack of information about the submillimeter/millimeter continuum prevents us from drawing any firm conclusion.

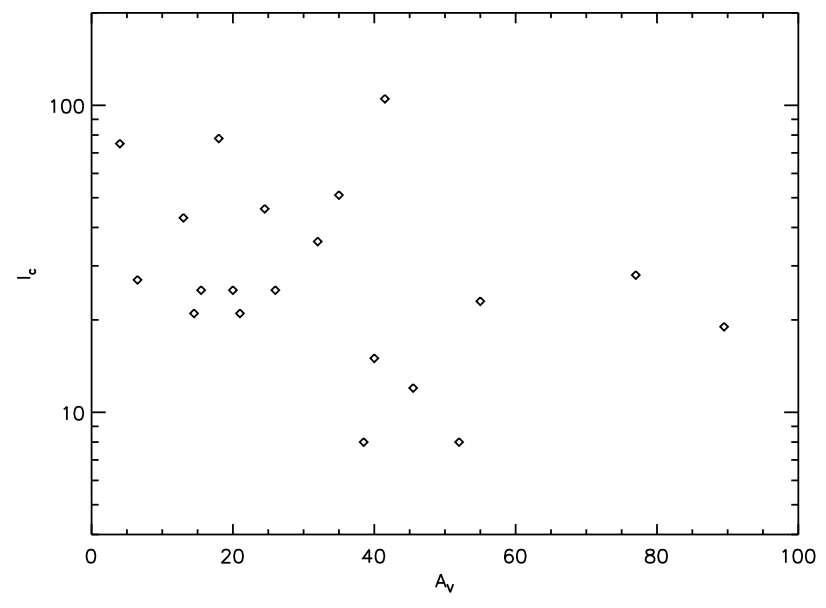

Fig. 2. Cluster richness indicator $I_{\mathrm{c}}$ as a function of $A_{V}$ at the cluster center; for a few detected clusters, we do not have an estimate of $A_{V}$.

Figure 2 shows $I_{\mathrm{c}}$ as a function of the peak $A_{V}$ and suggests that with higher dust extinction, we may find it more difficult, or it becomes less likely, to detect a cluster at $2.2 \mu \mathrm{m}$.

Our detection rate is quite high and this implies that young stellar clusters in sites of intermediate and massive star formation are ubiquitous. While this was established for relatively old Pre-MS systems such as Herbig Ae/Be stars (Testi et al. 1999), we hereby verify that this is also true in much younger systems, where the most massive stars may even be in a pre-Hot Core stage (Molinari et al. 2008a).

Our detection rate is higher compared to other similar searches of stellar clusters toward high-mass YSOs. For example Kumar et al. (2006) used the 2MASS archive and reported a rate of $25 \%$ (rising to $60 \%$ when neglecting the inner Galaxy regions) toward a larger sample, which also includes the sources of this work; in particular, we detect all clusters also detected by Kumar et al. and in addition we reveal clusters toward 13 objects for which Kumar et al. report no detection. The reason for this discrepancy may be because we obtained dedicated observations, while Kumar et al. used data from the 2MASS archive; the diffraction-limited spatial resolution of our data is between a factor of 4 and a factor of 10 better with respect to 2MASS, and this certainly facilitates cluster detection especially in particularly crowded areas such as the inner Galactic plane. To test this hypothesis, we degraded the NTT $K_{\mathrm{S}}$ image of Mol103, also considered in Kumar et al., to the 2MASS resolution; extraction and photometry were performed as outlined above but the search for a cluster based on the stellar radial density profiles revealed no cluster. The estimated number of members (corrected for the contribution of fore/background stars) for 7 out of the 10 clusters detected both by us and by Kumar et al. was at least a factor of two less in the latter study.

Kumar \& Grave (2008) conducted a similar study on a large sample of high-mass YSOs, that included some of our sources, using data from the GLIMPSE survey (Benjamin et al. 2003). They detect no significant cluster around any targets in a sample of 509 objects. As the authors say in their paper, however, GLIMPSE data are sensitive to $2-4 M_{\odot}$ pre-main sequence stars at the distance of $3 \mathrm{kpc}$. Based on color-magnitude analysis (see later below), our mass sensitivity is of the order of $1 M_{\odot}$ at a distance of $3.6 \mathrm{kpc}$ and $\sim 0.6 M_{\odot}$ at a distance of $2.1 \mathrm{Kpc}$. Probing longer wavelengths, GLIMPSE is likely to be more sensitive to younger sources compared to the classical $J, H, K$ range, which also samples relatively older pre-MS objects. The combination 


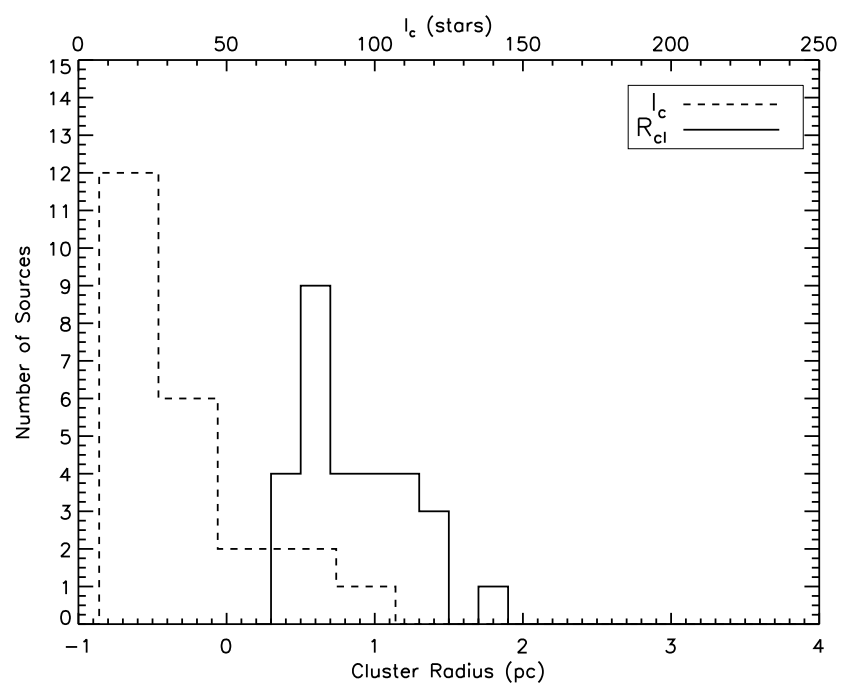

Fig. 3. Distribution of the cluster radii in parsecs (full line) and the cluster richness indicator $I_{\mathrm{c}}$ in number of stars (dashed lines); the median values for the two distributions are $0.6 \mathrm{pc}$ and 37 stars, respectively.

of sampling higher-mass (and hence rarer stars because of the shape of the IMF) and relatively younger stars (which, as indeed our analysis finds, may not be the majority in a young cluster) may plausibly be the reason for the negative cluster detection results of Kumar \& Grave.

The distribution of the radii of the detected clusters indicated by a full line in Fig. 3; the median value is $0.7 \mathrm{pc}$. The dashed histogram (which refers to the upper X-axis) shows the distribution of the cluster richness indicator $I_{\mathrm{c}}$, with a median number of stars of 27 . We note that the value of $I_{\mathrm{c}}$ for many of our clusters is less than the limit of 35 suggested by Lada \& Lada (2003) to be a bona fide cluster. This definition stems from the argument that a less rich agglomerate may not survive the formation process as an entity. Our interest, however, is to investigate the spatial properties of the young stellar population in a star-forming region at the time of active formation, without worrying about its possible persistence as a cluster at the end of the formation phase. However, we prefer not to introduce a new term to identify the structures that we see and still use the term cluster, although in a milder way than Lada \& Lada.

\subsection{Properties of identified clusters}

We first derive qualitative measurements related to the nature of the identified clusters using simple diagnostic tools such as color-color and color-magnitude diagrams. These diagrams have been drawn for all detected clusters and are available in electronic form; we illustrate here the particular case for Mol28.

\subsubsection{Color-color analysis}

Figure 4 shows the $[J-H]$ versus $\left[H-K_{\mathrm{s}}\right]$ diagram for all sources detected within a distance equal to $R_{\mathrm{cl}}$ centered on the stellar density peak. The full circles represent all sources detected in all three bands, the arrows representing sources with lower limits (to their magnitude) in the $J$ band. The plot shows more stars than the $I_{\mathrm{c}}$ value reported in Table 2 because we also include the fore/background stars that cannot be individually distinguished from the true cluster members. A significant fraction of the sources have colors compatible with main-sequence stars that have a variable amount of extinction reddening (computed

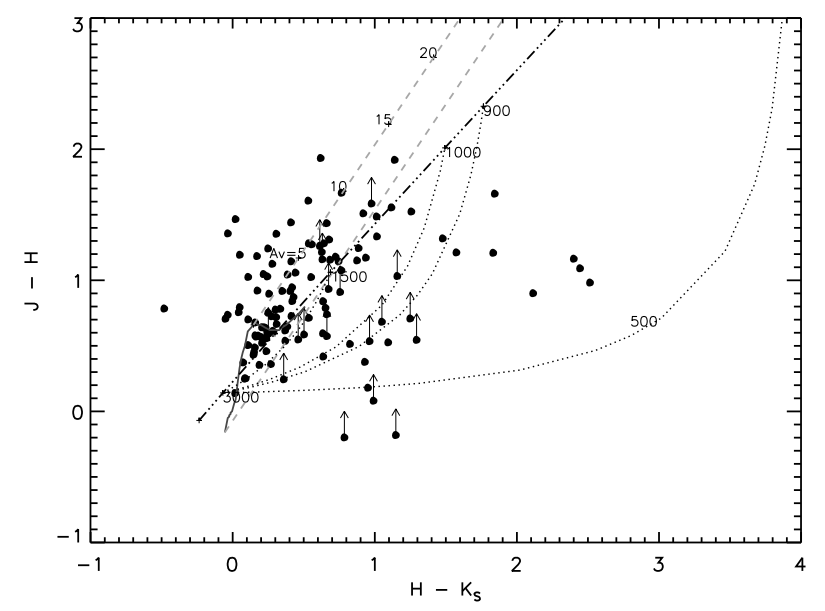

Fig. 4. $[J-H]$ vs. $\left[H-K_{\mathrm{s}}\right]$ diagram for Mol28. Upward pointing arrows are the sources not detected in $J$. The continuous curve at the bottomleft represents the main sequence, while the dashed grey lines represent the effect of reddening (Rieke \& Lebofsky 1985) for variable amounts of extinction as indicated along the lines. The dashed-dotted black line is the black-body curve, and the dotted curves are two-component black-body curves with varying relative contribution (respectively, from the inner to the outer curve, 3000-1500 K, 3000-1000 K, 3000-900 K and $3000-500 \mathrm{~K}$ ).

by adopting the Rieke \& Lebofsky (1985) extinction curve), but many sources have colors that are typical of young pre-MS objects with an intrinsic IR excess produced by warm circumstellar dust distributed in disks (Lada \& Adams 1992). The set of dotted curves represents the locus of two-component black bodies with temperatures as indicated at the start and end of each dotted line; along each curve, the relative contribution of the the two black bodies is varied. These curves mimic the effect of a temperature stratification in the dusty circumstellar envelopes, and the presence of sources in the area covered by these curves is an indication of the presence of warm circumstellar dust.

A straightforward indication of the youth of the cluster may be provided by the fraction of sources that are not compatible with being reddened MS stars, i.e., those with IR excess. The number of stars with an IR excess is normalized to the total number of stars detected in the cluster area, corrected for the expected number of fore/background stars estimated from the areas surrounding the cluster (but still in the same imaged field). To be conservative we extend the region of the MS by 0.2 mag to the right corresponding to about a $2 \sigma$ uncertainty in measured magnitudes. This ratio is reported as a percentage value in Col. 6 of Table 2.

\subsubsection{Color-magnitude analysis}

Additional evolutionary indications of the detected clusters may be derived from the $K_{\mathrm{s}}-\left[H-K_{\mathrm{s}}\right]$ diagram, reported for Mol28 in Fig. 5. Compared to the main sequence (the leftmost almost vertical curve in the figure) a significant fraction of the sources are on its right, where the evolutionary tracks for Pre-MS sources (Palla \& Stahler 1999) can also be found, and could therefore be interpreted as very young pre-MS objects. The distribution of sources in the diagram spans a much larger region than that covered by the Pre-MS isochrones, because of the combined effect of extinction reddening and IR excess. The extinction effects can be seen from the dotted lines originating in the main sequence and extending toward the bottom-right for increasing values of $A_{V}$. On the other hand, the presence of a warm dusty 


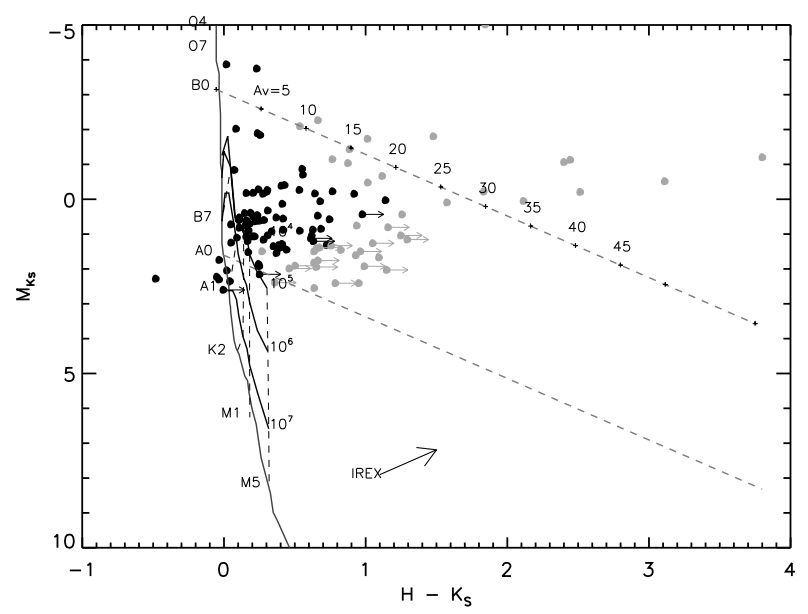

Fig. 5. $K_{\mathrm{s}}$ vs. $\left[H-K_{\mathrm{s}}\right]$ diagram for Mol28. The leftmost curve represents the main sequence, while the dashed lines represent the effect of reddening for variable amounts of extinction. Isochrones from Palla \& Stahler (1999) are also indicated with full lines for different Pre-MS ages. The arrow labeled IREX indicates the direction of change due to IR-excess (see Sect. 4.2). Rightward pointing arrows represent those sources not detected in $H$. Symbols in grey color indicate sources with IR excess as determined from the color-color diagrams (see Fig. 4).

circumstellar envelope implies an increase in both absolute emission and SED steepness, which would shift a pure photosphere toward the top-right of the diagram (as shown by the arrow labeled "IREX" in Fig. 5). In a similar way to the color-color analysis, it is impossible to estimate the age of individual stellar sources based on their location on the pre-MS isochrones, because we do not know the amount of $A_{\mathrm{V}}$ by which we should de redden each object. We follow a conservative approach by dereddening each object using half of the exctinction estimated for each location from millimeter maps; this corresponds to placing each object midway through the clump.

A further correction is to remove the IR excess for those sources, which is apparent in the color-color diagram (of Fig. 4), estimated using the formulation suggested by Hillenbrand \& Carpenter (2000), and used later in this work (see Sect. 4.2). The ratio of pre-MS stars to the total in each cluster area will remain contaminated by fore/background stars; to estimate this contamination, we choose an off-cluster area in the same imaged field and simply compute the ratio of sources with pre-MS colors to the total (in these off-cluster regions in which there is no significant reddening to correct for). for each cluster, Col. 7 of Table 2 reports the fraction of stars (detected in the cluster area in all three bands) situated more than $0.2 \mathrm{mag}$ to the right of the MS after the various corrections have been applied.

\section{Initial mass functions and star formation histories}

As is apparent from the qualitative analysis presented in the previous paragraphs, the diagnostic power of our observations is limited because we do not know which objects in the cluster area are true cluster members nor the precise amount of dust extinction (originating within the hosting clump) and IR excess (originating in the immediate circumstellar environment) pertaining to each source. Without this detailed knowledge of individual stars in the clusters, fundamental quantities such as the initial mass function (IMF) and the star formation history (SFH) cannot be derived directly from, e.g., the $K_{\mathrm{s}}$ luminosity function (KLF).
We are compelled to obtain these using statistical simulations of clusters based on different input parameters and performing a statistical comparison between synthetic and observed KLFs and $\mathrm{HKCFs}$.

We first derive the observed KLFs from the observations. We then illustrate in detail the model used for the cluster simulations, exploring the sensitivity of the results to a wide range of input parameters finally, modeled and observed KLFs are compared to infer statistically the IMF and SFH for our clusters.

\subsection{Observed $K_{\mathrm{s}}$ luminosity functions}

The KLF of each cluster is obtained by simply counting all detected sources within the cluster area as identified from the cluster density profile (see Sect. 3.1). In a similar way to the other diagnistic tools (Sects. 3.2.1 and 3.2.2), the KLF is contaminated by field stars that cannot be individually identified. To account for the field star contamination in a statistical way we subtract from the KLF that was compiled for the cluster area, the KLF for a region outside the cluster area but still in the same imaged field, after normalising the different areas. The regions in which the field star KLF is compiled have a lower extinction with respect to the cluster KLF, so the background contribution to the cluster KLF is likely to be overestimated. Field-subtracted KLFs for all clusters are presented in Appendix 6, and are also available online ${ }^{2}$.

The integral of the KLF provides an independent estimate of the number of cluster members, and these values are reported as $N_{\text {obs }}$ in Table 2. Their agreement with the richness indicator $I_{\mathrm{c}}$ confirms the consistency of our analysis. All KLFs show a dominant peak that is always close to the completeness limit, showing that our observations are insufficiently sensitive to the low-mass stellar component of our clusters. Many of the KLFs present a separate small peak at low magnitudes (one or two sources at most, on average). Could this be caused by confusion because of source crowding and insufficient spatial resolution? For each cluster, we studied the distribution of distances of each star from its nearest neighbour and found that there are two types of distributions, reported in Fig. 6. In the first type (full line in figure), the distribution has a peak corresponding to an inter-star distance significantly higher than the value corresponding to half the PSF FWHM (the full vertical line); in this case, the suggestion is that all cluster members have been resolved from their neighbour. In the second type (dashed line in the figure), the distribution has its peak very close to half the PSF's FWHM (the dashed vertical line), indicating that source blending should certainly be considered possible. We verified that all clusters with a distance distribution of the second type do exhibit a second faint peak at high brightness in their KLFs, therefore confirming that this feature is an artifact of the relatively low spatial resolution, which in some cases is insufficient to resolve all cluster members.

\subsection{Synthetic KLF. Synthetic cluster generator: a near-IR cluster simulator}

As already mentioned, we cannot derive masses and ages from our data alone. We thus developed a model to create statistically significant cluster simulations obtained for different assumptions of IMF and SFH (source ages and their distribution), and compare the synthetic KLFs with the observed field-subtracted

\footnotetext{
${ }^{2}$ At http://galatea.ifsi-roma.inaf.it/faustini/KLF/.
} 


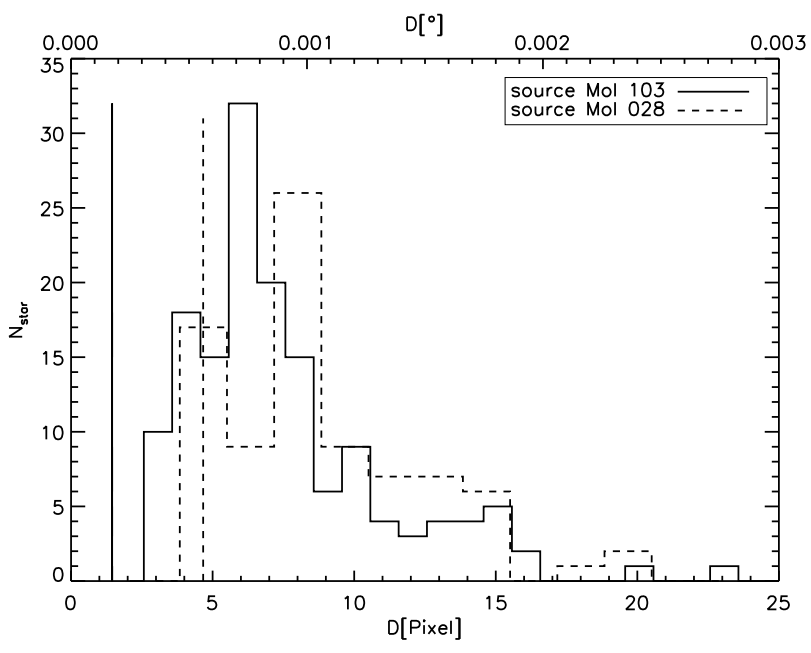

Fig. 6. Distribution of identified sources as a function of nearestneighbor distance $(D)$ for two of our examined fields (Mol28 dashed line and Mol103 full line).

KLFs. This model we called the synthetic cluster generator (SCG).

\subsubsection{SCG: model description}

A cluster is created by adding stars whose masses and ages are assigned via a Monte-Carlo extraction according to the chosen IMF and SFH; the pre-MS evolutionary tracks of Palla \& Stahler (1999) are then used to convert them into $J, H$, and $K_{\mathrm{s}}$ magnitudes. The $3 \mathrm{D}$ distribution of stars is obtained by randomly choosing for each star a set of $x, y, z$ coordinates using the observed stellar density profile (see Sect. 3.1), approximated to be a radially symmetric Gaussian, as weight-function; using submm continuum images, this is needed to assign the proper column of cold dust "required" to extinguish the near-IR radiation. Other analytical functions could have been used, e.g., a King profile, but the statistics of our clusters are insufficiently high to explore the effect of different radial profile assumptions.

To convert the submm flux into dust column density, we used the dust temperature and emissivity exponent $\beta$ determined in Molinari et al. (2000); mean values from the latter work were adopted for those fields not covered by our work.

To properly simulate the pre-MS stars, we also need to include the effect of an IR excess caused by warm dust in the circumstellar envelopes and disks. We used the distribution (modeled as a Gaussian) of $\left[H-K_{\mathrm{s}}\right]_{\mathrm{ex}}$ color excesses as measured for a sample of Pre-MS stars in Taurus, as used by Hillenbrand \& Carpenter (2000), as a weight-function to randomly assign a $\left[H-K_{\mathrm{S}}\right]_{\mathrm{ex}}$ to each simulated star in our model; the $K_{\mathrm{S}}$ vs. $\left[H-K_{\mathrm{s}}\right]_{\mathrm{ex}}$ relationship adopted in the above mentioned work was then used to derive the $H$ and $K_{\mathrm{s}}$ excess-corrected magnitudes. The $K_{\mathrm{s}}$ magnitude of the synthetic star was then compared with the limiting magnitude typical of the cluster being simulated to determine whether the star could have been detected in our observations. This procedure is repeated until the number of synthetic detectable stars equals the value of $I_{\mathrm{c}}$ determined for our observations; at this point, the cluster generation process is complete.

Since the simulation is based on Monte Carlo extraction of stellar mass, age, and position in the cluster, each independent run for a fixed set of input parameters can in principle result in very different outputs in terms of cluster luminosity, total stellar mass, maximum stellar mass, and synthetic KLF. To determine the statistical significance, the model is run 200 times for any given set of input parameters, and the median KLF is later adopted for comparison with the observed one. Clearly, the predictive power of this simulation model resides in its capability to characterize the cluster properties of any given parameter set. In other words, the distribution of the resulting quantities should not be uniform but peaked around characteristic values. We return to this point in Sect. 4.2.3

\subsubsection{SCG: input assumptions}

We tested three different assumptions about the star formation histories in our cluster simulations. The first was to assume that stars in the cluster formed in a single burst-like event (hereafter $\mathrm{SB}$ ) some $t_{1}$ years ago. The explored range in the simulations is $10^{3} \leq t_{1} \leq 10^{8} \mathrm{yrs}$. The second was that the formation of stars proceeds at a constant rate (hereafter CR) from a time $t_{1}$ years ago to a time $t_{2}$ years ago. The ranges explored in the simulations are $10^{4} \leq t_{1} \leq 10^{8} \mathrm{yrs}$ and $10^{3} \leq t_{2} \leq 10^{7} \mathrm{yrs}$, where we always assume that $t_{1}>t_{2}$. The third possibility that we explored was a variation in the previous assumption, where the star formation rate is not constant but varies with time as a Gaussian function (hereafter GR). Within the boundaries of the start and end of the star formation process, $t_{1}$ and $t_{2}$ that were varied as above, we also varied both the time $t_{\mathrm{c}}$ of the Gaussian peak in the range $10^{3.7} \leq t_{\mathrm{c}} \leq 10^{7.7}$ and $\log _{10}(\sigma)$ of the Gaussian-like SFH, which was allowed to have one of two values 0.1 and 0.5 .

We allowed three different choices of IMFs, i.e., Kroupa et al. (1993), Scalo (1998), and Salpeter (1955), with the latter modified by introducing a different slope for $M<1 M_{\odot}$ coinciding with that of the Scalo (1998) IMF; the three IMFs were labeled IMF1, IMF2, and IMF3, respectively. The IMF from Kroupa et al. provides a more accurate description of the lowmass end of the distribution, while the classical Salpeter IMF is flatter at low mass but heavier at intermediate and high masses (above $1 M_{\odot}$ ). The properties of the Scalo IMF is in-between the other two, resembling Salpeter's one below $1 M_{\odot}$ and above $10 M_{\odot}$, and Kroupa's for $1 M_{\odot}<M<10 M_{\odot}$.

\subsubsection{SCG: predictive power}

To verify our model's predictive power, we completed 200 simulations for a cluster with a Salpeter IMF and a constant star formation rate with $t_{1}=10^{6} \mathrm{yrs}$ and $t_{2}=10^{4} \mathrm{yrs}$. Figure 7 shows the distribution of the predicted number of stars and the total luminosity for the 200 simulations. The number of cluster members shows very little variation, as expected since the number of detectable stars is the parameter that we use to stop the simulation; on the other hand, the distribution of the total luminosity is not particularly peaked, as the central 3 bins containing about $60 \%$ of the simulations span almost two decades in luminosity.

On the other hand, the distributions for the total cluster stellar mass, and for the mass of the most massive member (see Fig. 8) are rather peaked and highlight a relatively higher predictive power of the model for these two quantities. It is to be noted that the distributions are rather skewed, suggesting that neither the mean nor the median are particularly suited to characterize the peak of the distribution. We indeed found that these quantities assume at their distributions peak a more representative value of mass and use them in the following discussion.

Concerning the reproducibility of the KLF, for each of the 200 runs the resulting KLF was fitted with a Gaussian function 


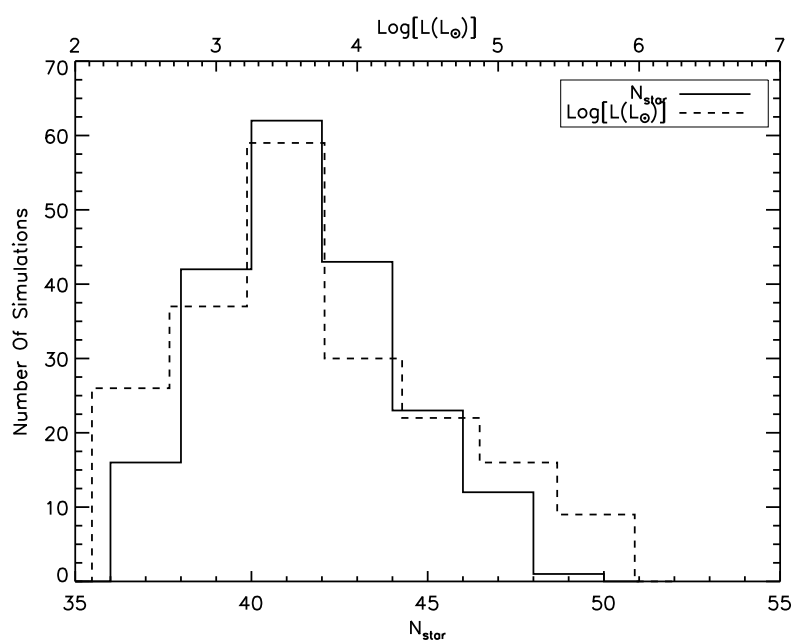

Fig. 7. Distribution of the predicted number of cluster members (full line) and total luminosity (dashed line) for 200 SCG runs for Mol160 with a Salpeter IMF and a constant star formation rate with $t_{1}=10^{6} \mathrm{yrs}$ and $t_{2}=10^{4} \mathrm{yrs}$.

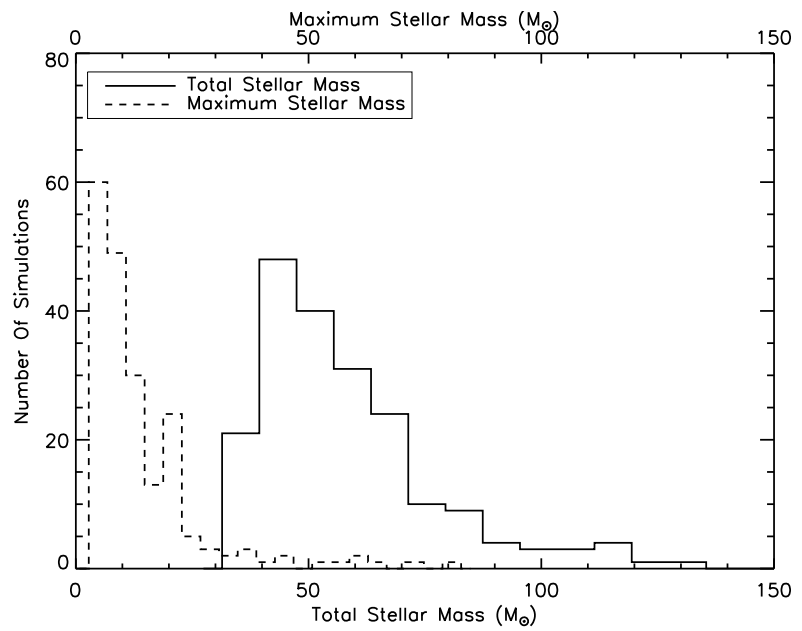

Fig. 8. Distribution of the predicted total stellar mass (full line) and mass for the most massive star (dashed line) in a cluster for 200 SCG runs for Mol160 (same inputs as in Fig. 7).

and the center, peak and $\sigma$ were determined. Figure 9 reports the distribution of these three parameters for the 200 runs and shows that all of them are remarkably peaked and symmetric. The formal rms spread for the three quantities, estimated via a Gaussian fit to the distributions in the figure, is $\approx 0.3 \mathrm{mag}$ for the KLF center, $\approx 12 \%$ for the KLF peak (about 1.2 sources out of a mean KLF peak of 10 ), and $\approx 0.25$ mag for the KLF FWHM.

We completed a similar analysis for HKCF $\left(H-K_{\mathrm{s}}\right.$ color function; see Sect. 3.2.2). Figure 10 shows the distribution of Gaussian function centers, peaks and $\sigma$ 's for HKCFs obtained for the same 200 runs used previously for the KLFs. Gaussian fits to the three distributions in the figure infer an rms that is $\approx 0.15$ mag for the HKCF center and $\approx 0.14$ mag for the HKCF $F W H M$, while the "peak" distribution is flatter and has an rms value of $\approx 21 \%$ for the HKCF peak (about 3.2 sources out of a mean $\mathrm{HKCF}$ peak of 15). It is worthwhile to stress that since the position that is assigned to each simulated star in the cluster is different in each of the 200 runs of the model (for any given set of input parameters), the scatter in the properties of the synthetic KLFs and HKCFs also statistically tends to account for the effects of extinction variations in the cluster's hosting clump,

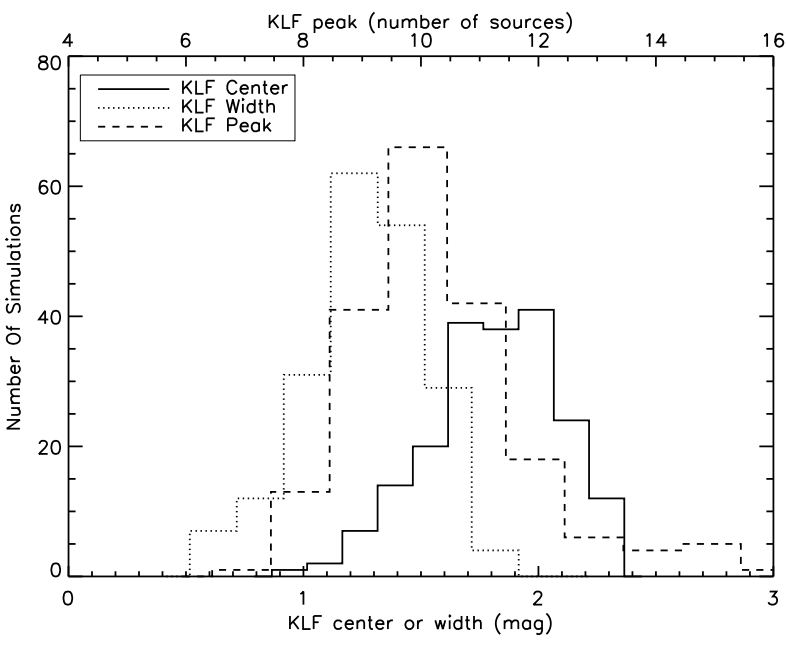

Fig. 9. Distribution of the predicted center magnitude (full line - bottom $\mathrm{X}$-axis scale), width (dotted line - bottom X-axis scale) and peak value (dashed line - top X-axis scale) of the predicted Gaussian-fitted KLFs for 200 SCG runs for Mol160 (same inputs as in Fig. 7).

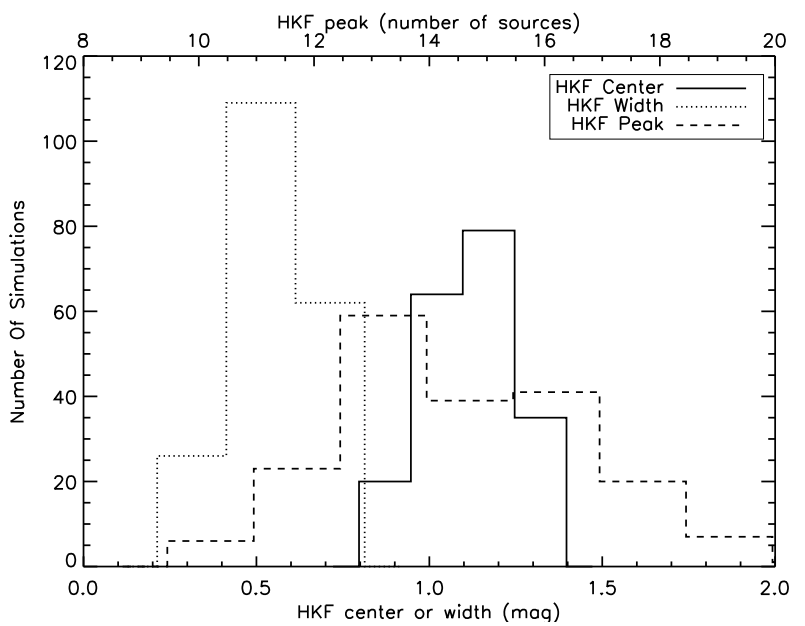

Fig. 10. Distribution of the predicted center color (full line - bottom $\mathrm{X}$-axis scale), width (dotted line - bottom $\mathrm{X}$-axis scale) and peak value (dashed line - top X-axis scale) of the predicted Gaussian-fitted HKCFs for 200 SCG runs for Mol160.

which may in principle be relevant in such heavily embedded systems (see Table 2).

For a given set of input parameters, we conclude that the model results, have a good reproducibility, except concerning the total luminosity. The model therefore has a strong predictive power concerning the median properties of a synthetic cluster. The spread in KLF center magnitudes is indeed, less than the bin amplitude used in compiling the KLFs for the simulations (and is used in the remainder of the work); the median synthetic KLF therefore provides a good representation of the cluster luminosity distribution.

In conclusion, 200 simulation runs for each combination of input parameters (IMF and SFH) can provide a robust assessment of the statistical significance of the synthetic observable properties (KLFs and HKCFs). Although the distributions for the KLFs' (HKCFs') parameters seem rather symmetrical, we adopt the median KLF (HKCF) of the 200 runs as a more reliable characterization for that particular parameters' set. The use of the mean KLF (HKCF) for the comparison does not significantly alter the results. 


\subsubsection{Exploring the SCG parameter space: cluster parameters}

After verifying the robustness of model results in independent runs for the same input parameters, we now measure the sensitivity of the model results to changes in these parameters. We first concentrate on simulated cluster physical parameters (number of cluster members, total luminosity, stellar mass distribution), and in the next paragraph we examine how the KLFs and the appearance of the color-magnitude diagrams, which are the main observables used in our analysis, behave in this respect.

Number of stars $N_{\text {stars }}-$ As a general rule, the older the cluster is allowed to be, irrespective of the detailed SFH adopted, the higher is the number of produced stars. This is easily understood since the SCG cluster formation is stops when the number of the $K_{\mathrm{s}}$-detectable stars equals the number of observed objects; if a cluster is old, the stars will be intrinsically fainter due to the shape of Pre-MS tracks and statistically less likely to extract stars bright enough to be detectable. As long as $t_{1} \leq 10^{6} \mathrm{yrs}$, $N_{\text {stars }}$ does not depend significantly on the IMF choice, while for older systems, IMF1 (Kroupa et al. 1993) produces nearly twice as many stars as IMF3 (Salpeter 1955) with IMF2 (Scalo 1998) in-between.

Stellar masses - Likewise, the total stellar mass and the mass of the most massive star will be higher the older the cluster is allowed to be. If an IMF1 cluster is a very old SB or a CR with $t_{1}=10^{8}$ yrs and $t_{2}=10^{7}$ yrs for example, $M_{\star \text { Tot }}$ and $M_{\star \text { Max }}$ will be respectively a factor of 5 and 2 higher than clusters that are younger and/or are allowed to form stars until recent times (i.e., allowing a CR with $t_{2}=10^{4} \mathrm{yrs}$ ). The explanation follows directly from the argument made for the $N_{\text {stars }}$ behavior above; matching the number of $K_{\mathrm{s}}$-detected stars in a relatively old cluster with intrinsically fainter stars will require that stars will have to be on average more massive objects, and this will clearly result also in a higher total stellar mass.

Going from IMF1 to IMF3, both $M_{\star \text { Tot }}$ and $M_{\star \text { Max }}$ significantly increase, as expected. The trend of $M_{\star \text { Tot }}$ with cluster age is less pronounced because with IMF2 and IMF3 it is statistically more likely to produce relatively more massive (and hence more easily detectable in $K_{\mathrm{s}}$ ) stars requiring a lower number of star extractions and hence a lower relative total mass at the end of the simulation. The age-trend of $M_{\star M a x}$ is instead the same (only shifted toward higher masses) because the probability of extracting a massive star is the same for all ages and is only a function of the chosen IMF.

Total stellar luminosities and massive object luminosities The total stellar luminosity, like the luminosity of the most massive star $\left(L_{\star \text { Max }}\right)$, exhibits the same behaviour as $M_{\star \text { Max }}$. This is easily understood given the steep power-law dependence of the stellar luminosity on mass, and confirms that the total luminosity $\left(L_{\mathrm{Tot}}\right)$ is largely dominated by the most massive stellar object in the cluster: $L_{\mathrm{Tot}} \propto L_{\star \mathrm{Max}}$. Of great interest is the ratio of $L_{\star \text { Max }}$ to $L_{\mathrm{Tot}}$; for the vast majority of clusters, its value varies between 0.6 and 0.8 . This is further confirmation that global properties of our clusters are dominated by the most massive source. This ratio does not present any particular dependence on the value of $M_{\star \mathrm{Max}}$, or of $N_{\text {stars }}$; only for the most populated clusters (clusters with of the order of a hundred members, such as Mol103, where the contribution of a great number of

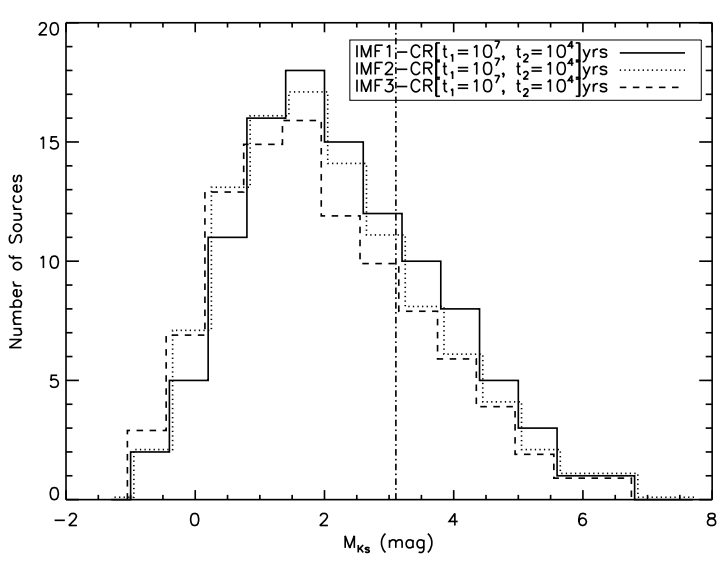

Fig. 11. KLF (using the absolute $K_{\mathrm{s}}$ magnitudes) for Mol3 predicted by SCG for a CR cluster with $t_{1}=10^{7}$ yrs and $t_{2}=10^{4} \mathrm{yrs}$, for three different choices of the IMF (line styles as indicated in the figure). The dash-dotted line represents the completeness limit for this source given the $K_{\mathrm{s}}$ limiting magnitude.

low-mass sources becomes important do we find a lower value for this ratio.

\subsubsection{Exploring the SCG parameter space: KLF variations}

We now briefly analyze the diagnostic power of the KLF and the HKCF against changes in IMF and SFH choices. Figure 11 shows the KLFs predicted for source Mol3 adopting the same SFH parameters (as indicated in the figure) and using the three different IMF choices.

The shape of the resulting KLF changes throughout the $M_{K}$ range; going from Kroupa et al.'s IMF1 to Salpeter's IMF3, the distribution becoming more skewed toward lower magnitudes; this was expected since IMF1 produces more lower mass stars than IMF3. One can certainly argue that the change is not dramatic, but on the other hand the modification does not affect one or two bins but the entire KLF consistently. The change is more apparent in the region between the peak and the completeness limit than at the bright end of the KLF, and for this reason the ability of the model to discriminate between different IMFs is higher for those sources, as Mol3 in the figure, where the KLF's peak is clearly detected above the completeness limit.

The difference in predicted KLFs is much more dramatic if different age ranges are assumed, while keeping fixed the shape of the SFH and the IMF, as it is apparent in Fig. 12. The peak of the KLF shifts considerably toward higher magnitudes as the median stellar ages $\left(t_{\mathrm{c}}\right)$ increase. A similar trend is observed by comparing SB models with different ages, although SB models always produce KLFs that are considerably narrower than CR or GR models. Older cluster ages would shift the peak of the KLF beyond the completeness limit; in other words, our analysis is insensitive to ages for the majority of stars in excess of $\sim 5 \times$ $10^{6} \div 10^{7} \mathrm{yrs}$; these old cluster ages would be hard to justify given that they are still heavily embedded in dense clumps.

Finally, we briefly show how the KLFs change for different choices of the SFHs. Figure 13 shows the KLFs obtained for a SB with $t_{1}=10^{6} \mathrm{yrs}$, compared with a CR with $t_{1}=10^{7} \mathrm{yrs}$ and $t_{2}=10^{4}$ yrs and a GR with the same start and end star formation period, and with a peak times for star formation rate of $t_{\mathrm{c}}=10^{6} \mathrm{yrs}$. The KLFs are clearly different, with a peak 


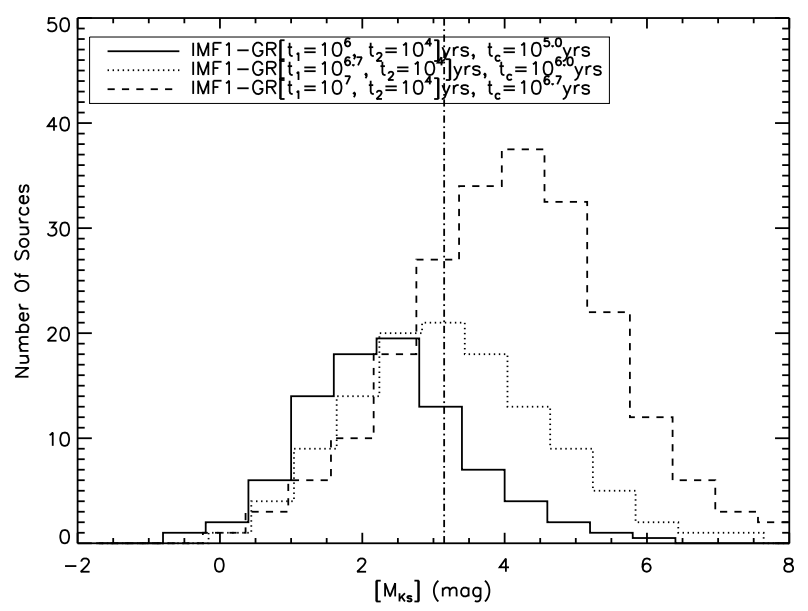

Fig. 12. KLF (using the absolute $K_{\mathrm{s}}$ magnitudes) for Mol3 predicted by SCG for an IMF3 cluster with different ages as indicated in the plot. Older clusters produce a KLF peaked tower lower magnitudes. The dash-dotted line represents the completeness limit of this source given the $K_{\mathrm{s}}$ limiting magnitude.

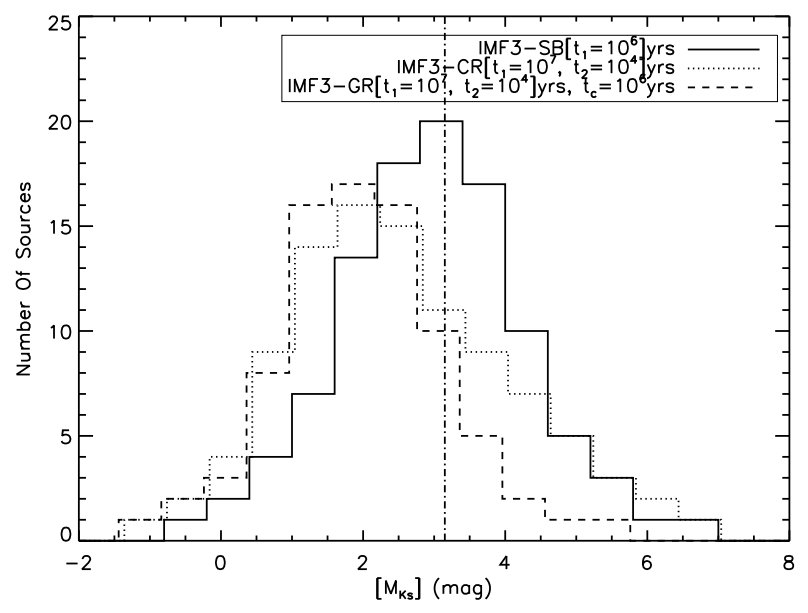

Fig. 13. KLF (using the absolute $K_{\mathrm{s}}$ magnitudes) for Mol3 predicted by SCG for an IMF3 SB cluster with $t_{1}=10^{6} \mathrm{yrs}$ (full line), CR with $t_{1}=10^{7}$ yrs and $t_{2}=10^{4} \mathrm{yrs}$ (dotted line), and GR with same $t_{1}$ and $t_{2}$ and $t_{\mathrm{c}}=10^{6} \mathrm{yrs}$ (dashed line). The dash-dotted line represents the completeness limit for this source given the $K_{\mathrm{s}}$ limiting magnitude.

magnitude which is quite sensitive to the formation rate typology and the peak time for star production.

The $\left[H-K_{\mathrm{S}}\right]$ color functions are found to be insensitive to the choice of IMF. As for the KLFs, the main differences between synthetic HKCFs are more evident for different age ranges especially in the number of detectable stars.

\subsection{Comparing observed and synthetic KLFs and HKCFs}

The detailed comparison of the model KLFs and HKCFs functions to those observed was carried out only for those sources where the number of detected stars was sufficient $\left(I_{c} \geq 15\right)$ to allow a statistically significant comparison (see Col. 3 of Table 2), and where submm information was available to allow meaningful estimates of extinction (this excludes Mol15 and Mo199). The number of clusters fulfilling these criteria were 16 out of 23 detected clusters. The comparison of the observed KLFs and HKCFs $\left(\mathrm{KLF}_{\mathrm{Obs}}, \mathrm{HKCF}_{\mathrm{Obs}}\right)$, with the synthetic ones produced by $\mathrm{SCG}\left(\mathrm{KLF}_{\mathrm{Syn}} \mathrm{HKCF}_{\mathrm{Syn}}\right)$, for the full set of input parameters
(IMF, SFH and age parameters) was carried out automatically; to ease the process, the observed and synthetic functions were computed on the same $M_{K}$ and $H-K$ grid.

The comparison procedure between synthetic and observed KLFs is described below, but it is the same for HKCFs. The KLFs are first compared bin by bin (the comparison being limited to those bins brighter than the completeness limit) identifying with $i$ each bin of the observed KLF, starting from $i=1$ for the lower- $M_{K}$ non-zero bin to $N$ for the bin where the completeness limit for that source is reached (the number $N$ differs clearly for each cluster). In the case of HKCFs, we exclude objects with $H$ and $K$ magnitudes brighter than the observed limiting magnitudes for these bands. A matching flag $m_{i}$ is set to be 1 for those bins where the number of sources coincide within the $1 \sigma$ Poissonian error bar of the observed KLF, i.e.,

$\left|N_{\star \operatorname{Syn}_{i}}-N_{\star \mathrm{Obs}_{i}}\right| \leq \sqrt{N_{\star \mathrm{Obs}_{i}}}$.

The total number of bins where a match is found is divided by the total number of bins useful to the comparison to obtain a KLF compatibility figure (in \%) of

$$
C=100 \times \frac{\sum_{i=1}^{N} m_{i}}{N} .
$$

The higher is $C$, the closer is the overall match between $\mathrm{KLF}_{\mathrm{Obs}}$ and $\mathrm{KLF}_{\text {Syn }}$.

However, the same value of $\mathrm{C}$ may result from bins concentrated at the low- $M_{K}$ end of the KLF, where there are few sources, or in the region around the peak and in the proximity of the completeness limit, where instead there are more sources and hence higher statistical significance. A further figure of merit is then introduced,

$W=\sum_{i=1}^{N} \frac{m_{i} \cdot N_{\star \mathrm{Obs}_{i}}}{N_{\star \mathrm{Obs}_{\mathrm{tot}}}}$,

where $N_{\star \mathrm{Obs}_{\text {tot }}}$ is the total number of sources present in all the bins used for comparison. This parameter weights each matching bin by its relative richness, favoring the bins closer to the completeness limit and the KLF peak over those in the bright tail of the KLF, and favoring for HKCF the bin closer to the peak of the distribution. This choice is because the KLF (HKCF) peak region is the most sensitive to changes in the SCG input parameters.

In this automatic procedure, we select models for which the parameters $\mathrm{C}$ and $\mathrm{W}$ (at the same time for KLFs and HKCFs) are maximum. For Mol8B, Mol45, and Mol84, the observed KLF has a very irregular and multiple-peaked shape that cannot be matched by any model, and are therefore discarded from further considerations. We are then left with 14 clusters for which a series of models can be found with at least $75 \%$ of the bins matching the observations. We find that the best values of $\mathrm{C}$ and $\mathrm{W}$ are never found for one single set of parameters, but rather we identify ranges of parameter values that produce the best match; in other words, there is a level of degeneracy that the models cannot remove, and this varies from source to source. In 4 clusters (Mol109, 110, 136 and 151), this degeneracy is essentially complete and the model is unable to make any prediction; in one case (Mol148), the comparison selects models with very old stellar ages but with total stellar luminosities by far in excess of the measured bolometric luminosity obtained by integrating the observed luminosities for this region from the mid-IR to the millimeter (see Table 2). In the 9 remaining cases, some degeneracy persists especially in the IMF, confirming (Sect. 4.2.5) that 
Table 3. Results for SCG runs on detected clusters.

\begin{tabular}{lccccccc}
\hline \hline $\begin{array}{l}\text { Source } \\
\text { Mol }\end{array}$ & IMF & $t_{10 \%}$ & $\begin{array}{c}t_{\text {med }} \\
\text { yrs }\end{array}$ & $t_{90 \%}$ & $N_{\star}$ & $\begin{array}{c}M_{\star \text { Max }} \\
M_{\odot}\end{array}$ & $\begin{array}{c}M_{\star \text { Tot }} \\
M_{\odot}\end{array}$ \\
\hline 3 & 1 & $10^{4.98}$ & $10^{5.40}$ & $10^{5.81}$ & 91 & 3.7 & 61 \\
$8 \mathrm{~A}$ & $2-3$ & $10^{4.71}$ & $10^{5.38}$ & $10^{5.83}$ & 31 & 3.8 & 30 \\
11 & $1-2-3$ & $10^{6.06}$ & $10^{6.41}$ & $10^{6.82}$ & 48 & 3.8 & 47 \\
28 & 3 & $10^{6.03}$ & $10^{6.12}$ & $10^{6.24}$ & 77 & 9.9 & 105 \\
50 & 1 & $10^{6.16}$ & $10^{6.48}$ & $10^{6.66}$ & 53 & 3.5 & 36 \\
103 & $1-2$ & $10^{6.57}$ & $10^{6.70}$ & $10^{6.83}$ & 115 & 4.0 & 80 \\
139 & 3 & $10^{5.34}$ & $10^{5.96}$ & $10^{6.46}$ & 25 & 2.9 & 16 \\
143 & 2 & $10^{6.57}$ & $10^{6.70}$ & $10^{6.82}$ & 27 & 3.1 & 21 \\
160 & $1-2-3$ & $10^{6.57}$ & $10^{6.7}$ & $10^{6.83}$ & 89 & 4.3 & 63 \\
\hline
\end{tabular}

our models are weakly sensitive to the IMFs, but there are clear indications concerning the $\mathrm{SFH}$ and ages.

Table 3 reports a summary of the results. The IMF of matching classes of synthetic cluster models is shown in Col. 2. Columns. $3-5$ contain the times for the formation of $10 \%, 50 \%$ and $90 \%$ of the total number of cluster members; these values are the median of the values that these times have in all models that match the observations. Column 6 is the number of cluster members $N_{\star}$, Col. 7 shows the mass of the most massive object $M_{\star \mathrm{Max}}$, and Col. 8 reports the total stellar mass of the cluster $M_{\star \text { Tot }}$. We emphasize again that the analysis selects classes of models other than single models; the values reported in Table 3 are the median value of the parameters for each class of matching models. The table shows that for some fields multiple IMFs are compatible with the data and, in general, SFHs with constant (CR) or Gaussian (GR) star-formation rates provide acceptable solutions for certain age ranges (as reported in the table). Simulations of SFHs with a single burst are, in general, not accepted. Our modeling is insensitive to bulk stellar ages in a cluster in excess of $5 \times 10^{6} \div 10^{7}$ years (Sect. 4.2.5).

\section{Discussion}

\subsection{Cluster ages and star formation histories}

Perhaps the most important result of this work is that in all clusters where the comparison of observed KLFs with the ones predicted by the SCG model is possible (see previous paragraph), the observations are consistent with a star formation that continues over time intervals that in most cases have a duration of between about few $10^{5}$ and few $10^{6} \mathrm{yrs}$, and with a median cluster age of a few $10^{6}$ yrs. In most cases, we cannot discriminate clearly between a constant or variable SFR but we are confident that we can exclude the possibility that on average the stars in our clusters are coeval and originate in a single burst of formation. Detailed studies toward the Orion Nebula Cluster indicate that stars have been forming for at least $10 t_{\mathrm{dyn}}$, or $20 t_{\mathrm{ff}}$ (Palla \& Stahler 1999; Hoogerwerf et al. 2001; Hillenbrand 1997), and our results would seem to generalize this on a larger sample of intermediate and high-mass star-forming regions.

In principle it can be argued that our analysis is incomplete since we did not consider longer wavelength data, which could identify heavily extincted objects that are barely visible, or not visible at all, in the near infrared. However, this does not modify appreciably our conclusions about the age spread within the clusters. Vig et al. (2007) indeed, applied a different analysis to the specific region Mol075, a field not included in our final analysis (Table 3 ) because the background-subtracted KLF is populated by too few objects for a statistically significant model comparison. Vig et al. also considered Spitzer IRAC and MIPS data, looking for the brighter and redder objects in the area covered by submillimeter emission. In this way, they could identify the younger and more massive objects in the field with an estimated age of the order of $10^{6}$ years or less. This approach, however, is insensitive to low mass and relatively older pre-MS objects, for which our method is ideally designed. While for this particular field, for the reasons explained above, we cannot perform a direct comparison to our approach, it is clear that the inclusion of longer wavelength data in the analysis might have identified a different, younger, population of objects, rather than increasing the observed age spread deduced for the clusters.

Models of cluster formation by competitive accretion appear to produce an IMF close to those observed because of thanks to the spread in the accretion rates of the competitive accretion mechanism. However, the prediction that all stars are formed in about $5 \times 10^{5}$ yrs (Bonnel et al. 2004) for typical conditions in young clusters, corresponding to a dynamical time or so, seems to disagree with our results. We instead favour scenarios (Tan \& McKee 2002) in which stars continue to forming over several free-fall times thus providing the required age spread. The finding that the most massive object in the fields considered in this work are still being formed or have just finished a phase of intense accretion (Molinari et al. 2008a) is a further indication that star formation seems to be a long-duration process in the life of a molecular clump.

How do our clusters compare to more evolved systems, such as the sample of Herbig Ae/Be stars observed by Testi et al. (1997, 1998)? Figure 14 shows the relationship between the mass of the most massive source and the total number of cluster stars as provided by the SCG simulations for our modeled clusters. The filled symbols represent the clusters that we could model (Table 3); the empty symbols instead represent the clusters that could not be modeled for a variety of reasons (see Sect. 4.3), while Testi et al.'s clusters are reported as asterisks (see figure caption for detailed explanation of the symbols). The figure suggests that the clusters presented in this study are richer than those surrounding Herbig Ae/Be stars for any given value of the most massive star in each cluster. The trend persists if we use similar indicators (e.g,. $I_{\mathrm{c}}$, the full triangles in the figure). Furthermore, we note that while the limiting magnitudes of our observations and those of Testi et al. (1998) are similar, higher $A_{V}$ values toward our sources and the typically greater distance from the Sun would justify the non-detection of the fainter cluster members predicted by the SCG models. It is thus likely that the values of $I_{\mathrm{c}}$ derived from our observations tend to underestimate the cluster memberships.

This evidence is clear for values of the most massive star in the cluster below $\sim 10 M_{\odot}$, where the 9 clusters for which we could compare observations with SCG predictions lie (the diamonds). In the clusters for which we haven't simulation results (the empty triangles), the mass for the highest-mass star was estimated by assuming that a fraction of between 30 and $100 \%$ of the bolometric luminosity originates in a single ZAMS star. In this case the trend toward richer clusters than Herbig stars (i.e., the asterisks) would become marginal. These estimates, however, place the latter clusters systematically to the right in the plot, compared to the 9 modeled clusters; indeed, if we were to estimate in the same way a maximum stellar mass also for the 9 modeled clusters, we would obtain values in excess (between a factor two and three) of those provided by the detailed SCG modeling. In other words, the evidence that our clusters are richer than those around Herbig stars is marginal at worst (i.e., using the most conservative approach of estimating the mass of the highest-mass star). 


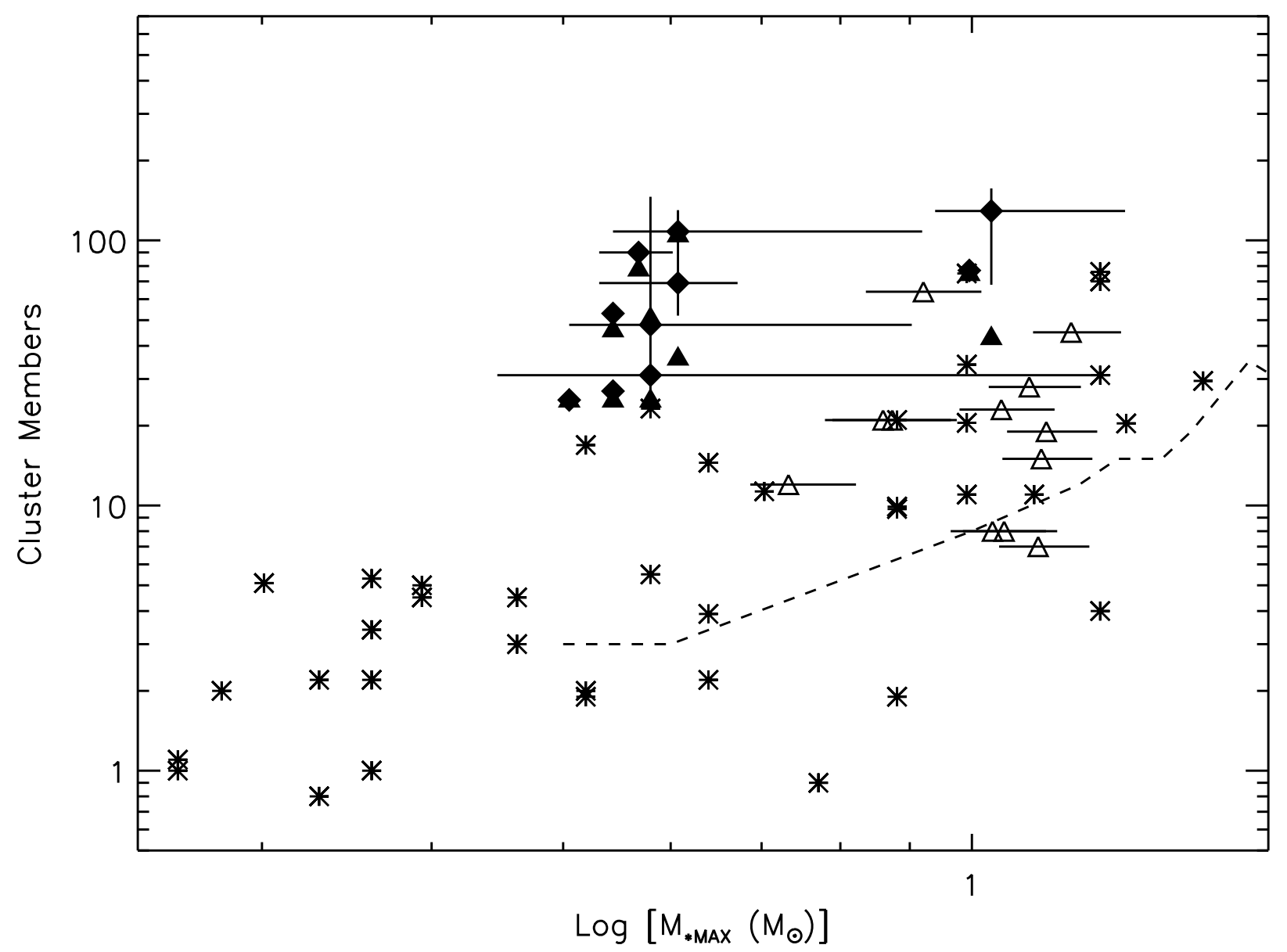

Fig. 14. Number of cluster members as a function of mass of the highest mass star. Asterisks are for Testi et al.'s (1997, 1998) Herbig Ae/Be sample. Other points are for our source sample, where the full diamonds are the $N_{\star}$ obtained from our cluster simulation analysis; and the full lines passing through the full diamonds represent the total spread in the parameters from all SCG models that match the observed KLF and HKCF. Full triangles are the same clusters with the observationally derived $I_{\mathrm{c}}$ instead of the model-provided $N_{\star}$. Empty triangles are those clusters that were not analyzed with SCG, or exhibit complete model degeneracy; in these cases $I_{\mathrm{c}}$ was used for the cluster membership (from Table 2), while the maximum stellar mass has been derived by assuming that half of the bolometric luminosity is generated by a single ZAMS star (the horizontal lines through the empty triangles represent the mass spread assuming that a fraction of between $30 \%$ and $100 \%$ of $L_{\text {bol }}$ comes from a single star). The dashed line delimits the region where $25 \%$ of the clusters randomly extracted from the IMF would follow in the statistical models (Testi et al. 2001).

The plausibility of this interpretation is strengthened by the results of Baumgardt \& Kroupa (2007) who completed extensive numerical simulations of the evolution of stellar clusters as a function of, among other parameters, the cluster gas content. They show that for a wide range of initial conditions and star formation efficiencies, the dispersal of the gas with age causes the cluster to expand overall and disperse a fraction of the stars originally belonging to the cluster. As the cluster expands, its decreasing stellar density ensures that the low-density outer regions of the cluster become ever more difficult to detect against the field stars (especially in the Galactic plane, where all these objects lie), mimicking a smaller cluster from an observational viewpoint. Figure 15 shows the distribution of radii for our clusters (full line) and for those surrounding Ae/Be stars; the radii were derived by the same analysis in the two samples and the histogram clearly shows that our clusters are indeed larger in size, confirming the prediction of Baumgardt \& Kroupa (2007).

This age effect on cluster size is also revealed within our clusters sample. Figure 16 presents the relationship between the cluster radii derived from the observations and their ages derived from the modeling. The reported correlation has a Spearman rank correlation coefficient of $\sim-0.6$, indicating a good

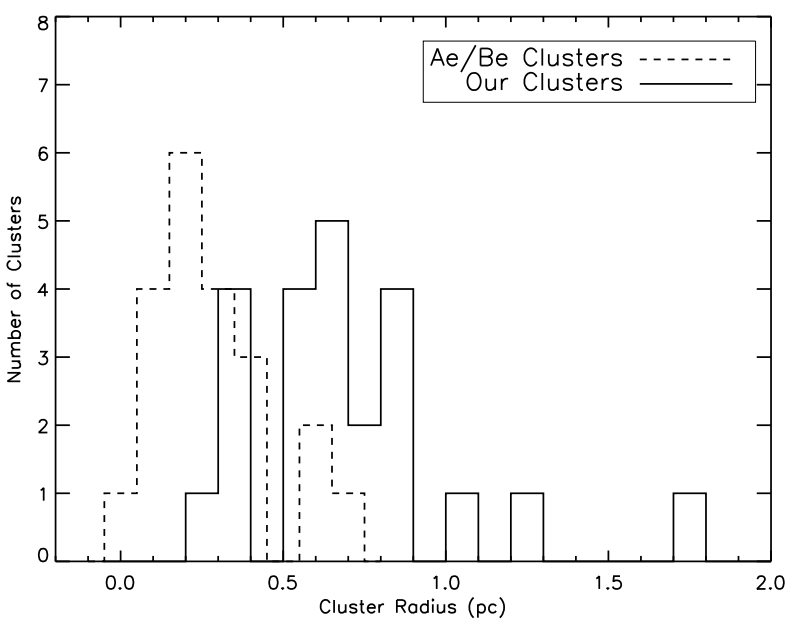

Fig. 15. Distribution of radii for our clusters (full line) and those associated with Herbig Ae/Be stars (from Testi et al. 2001, dashed line).

correlation with a significance of $92 \%$ (between 2 and $3 \sigma$ ). Ongoing gas dispersal is certainly plausible in our clusters, given 


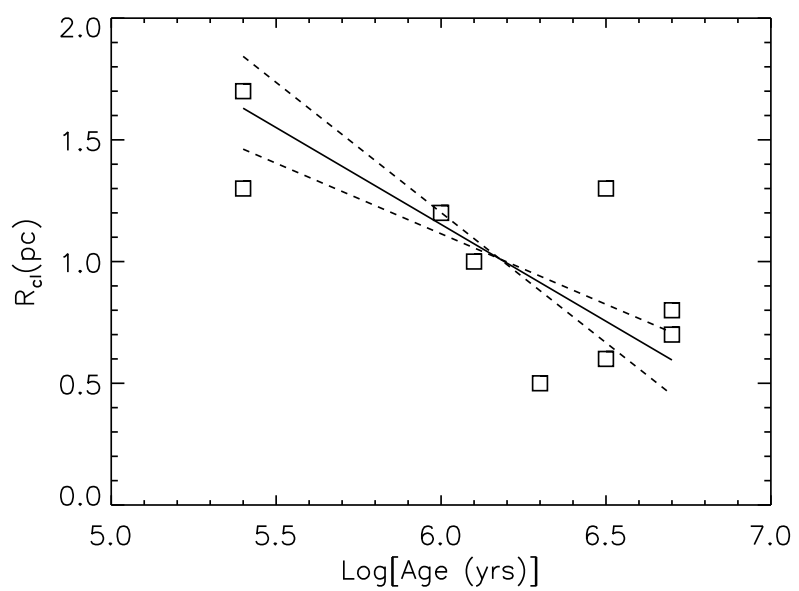

Fig. 16. Cluster radii (from Table 2) as a function of the cluster median ages (from Table 3). Dashed lines represent the linear fits obtained fitting in turn one plotted variable as a function of the other; the full line is the bisector of the two dashed lines and represents the fit which minimizes the quadratic geometric (i.e. not along the $\mathrm{X}$ or $\mathrm{Y}$ axis alone) distance of the data from the fit. Following Isobe et al. (1990), this is the adequate approach when the nature of the data scatter around the linear fit is not known (and it is not due to classical measurement uncertainties); the slope of the full line is $-0.8 \pm 0.2$ and the $1 \sigma$ spread is within the area enclosed by the two dashed lines.

the common detection in these systems of molecular outflows (Zhang et al. 2001, 2005), which are highly effective in transferring material away from the forming objects and possibly out of the star-forming region; parsec-scale flows are also commonly observed from low-mass YSOs.

The final stage of gas dispersal, eventually leading to optically revealed clusters such as those around $\mathrm{Ae} / \mathrm{Be}$ stars, might be triggered by the powerful winds and radiation fields from newborn $\mathrm{O}$ and $\mathrm{B}$ stars. The indications are (Molinari et al. 2008a,b) that the most massive objects forming in the densest regions of the clumps hosting our clusters may not yet have reached the ZAMS, or are just starting to develop their HII regions. It is quite likely that this event will mark the moment of maximum efficiency of gas dispersal and further evolution of our clusters toward the Ae/Be's ones.

\subsection{Physical vs. statistical models for cluster formation}

Figure 14 can also be used as a diagnostic to discriminate between different classes of models for the origin of clusters. Testi et al. (2001) called physical the class of models that implies a physical relationship between the most massive star that forms and other environmental properties such as the cluster richness or the mass of the gaseous clump where the stars originate from; examples are the "turbulent core" (McKee \& Tan 2003), the "coalescence" (Bonnell et al., 1998), or the competitive accretion models (Bonnell \& Bate 2006). In a second class of models, called statistical, the relationship between the most massive star in a cluster and its richness originates in the higher probability of finding the rare massive stars in rich clusters rather than in isolation (Bonnell \& Clarke 1999). If clusters are populated by randomly picking stars from the field stars' initial mass function, and considering a cluster membership-size distribution in the form of an appropriate power law, then the observations of Testi et al. (1999) can be naturally explained. Nevertheless, this model predicts that a significant fraction of high-mass stars are still associated with relatively poorly populated clusters, in other

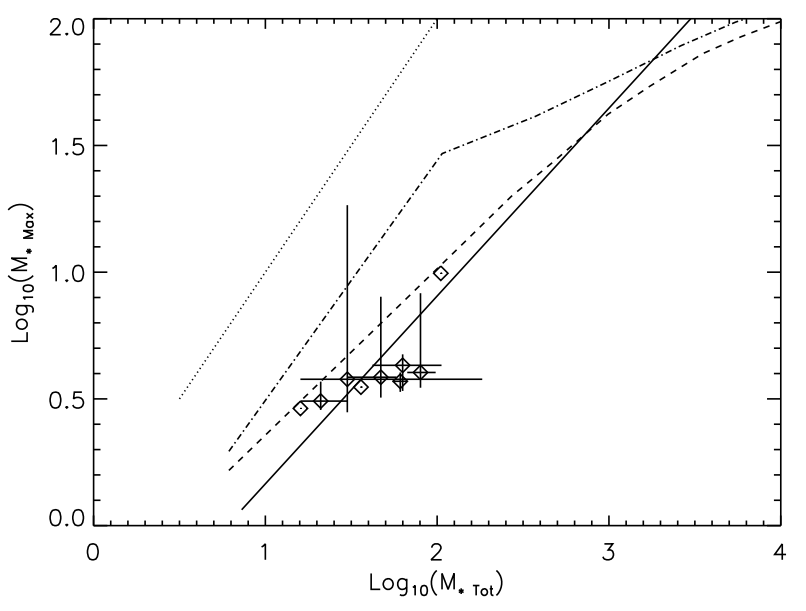

Fig. 17. Mass for the highest mass star as a function of the total stellar mass for the 9 modeled clusters (see Table 3); the bars associated to each cluster (the diamond symbols) represent the total span of the parameter values for the classes of models selected by our analysis (the diamond marks the median values). The dash-dotted, and dashed lines represents the relationship obtained for numerical simulations of clusters drawn from pure random sampling of the IMF, and using a so-called sorted sampling, following Weidner \& Kroupa (2006). The full line is a semianalytical approximation of this relationship obtained by Weidner \& Kroupa (2004). The dotted line is the limit where a cluster is made of just one star.

words that massive stars can be found both in high- $N$ clusters and, to a lesser extent, in low- $N$ clusters.

The dashed line in Fig. 14 is the upper boundary of the region that should contain $25 \%$ of the cluster realizations obtained by randomly extracting stars from the IMF (Testi et al. 2001). If we consider our measurements of $I_{\mathrm{c}}$ for our entire sample (i.e., the full and empty triangles), a fraction of about $15 \%$ of the clusters is found marginally below the dashed line. However, we note that our modeling was possible only for clusters above a membership threshold derived with $I_{\mathrm{c}}$, which is thus a biased subsample toward rich clusters. Based the extreme assumption that the fields with no detected cluster are cases of systems consisting of a single heavily extincted star, and thus would fall below the dashed line in Fig. 14, this fraction would approach the $25 \%$ level. This, however, is an extreme case because, as we have already discussed, the high value of the extinction derived from submillimeter maps may be the reason for not detecting clusters in at least a number of observed fields.

As an additional means of differentiating between physical and statistical cluster models, Weidner \& Kroupa (2006) argued that a non-trivial correlation exists between the highest-mass star in a cluster, $M_{\star \mathrm{Max}}$, and the total stellar mass of the cluster itself, $M_{\star \text { Tot }}$ (Fig. 17). Numerical simulations show that the relationship obtained by pure random sampling of the IMF with an imposed physical limit of $150 M_{\odot}$ for the maximum stellar mass (the dashed-dot line in Fig. 17) clearly does not represent our data.

A substantially different result (the dashed line) is obtained if cluster members are selected in ascending order and constrained to total cluster masses distributed according to a cluster total mass function (Weidner \& Kroupa 2006). Basically, this second option infers that drawing 10 clusters of 100 stars will not deliver the same $M_{\star \mathrm{Max}}=f\left(M_{\star \mathrm{Tot}}\right)$ as drawing 1 cluster of 1000 stars. This trend closely resembles a semi-analytical approximation of $M_{\star \text { Max }}=f\left(M_{\star \mathrm{Tot}}\right)$ obtained by Weidner \& Kroupa (2004), again assuming that total cluster stellar masses 
are distributed according to a power-law mass function. Weidner \& Kroupa (2006) suggested that this sorted sampling way of populating a cluster can be physically understood in terms of a pre-stellar clump where initial low-amplitude perturbations start low-mass star formation; as further perturbations with larger amplitude grow, higher mass stars will start to form until the feedback from the latter will begin to disrupting the cloud. This scenario of organised star formation where low-mass stars are the first to form, is the same as we favor (see Sect. 5.1) considering the age spread that we find in our clusters in which, based on independent considerations (Molinari et al. 2008a), the most massive star may not have formed. By the way, this latter possibility does not change the substance of the agreement between our data and the physical cluster models in Fig. 17, since the late addition of the highest mass star currently not yet visible in the near-IR would shift the points toward the top-right of the plot.

We verified a posteriori that the range of $M_{\star \text { Max }}$ and $M_{\star T o t}$ parameters values explored by our simulations is much wider than the area spanned by the bars attached to the single points, and also includes regions compatible with the random sampling cluster model. We then conclude that the result of Fig. 17 is not likely to be produced by a biased sampling of the clusters' physical parameters explored in our models.

The predictions of the sorted sampling descibed above, with which our data points best agree, are also in good agreement with the results from simulations of clusters forming in a competitive accretion scenario (Bonnell et al. 2004). This model, however, seems to be excluded by the observed ages and age spreads in our clusters which are in clear disagreement with the predicted cluster formation timescales of 2-3 free-fall times.

\subsection{Influence of binarity on the interpretation of age spread}

Weidner et al. (2008) carried out extensive numerical simulations to determine how the presence of unresolved binary/multiple stars can affect the observational properties of a young cluster in a massive star-forming region. Assuming 100\% binarity in a cluster of coeval sources, they find that unresolved binaries may lead an observer to conclude that a significant age spread is instead present in the cluster; the full line in Fig. 18 shows the a posteriori age determination assuming that all binaries are unresolved. We see that the measured age spread for the large majority of stars simulated in Weidner et al.'s simulation is comparable to a $\log ($ age $)$ Gaussian distribution with $\sigma=0.1$, which is one of the possible choices of star formation histories in our cluster models. However, the comparison of our observed KLFs with the SCG models (Table 3) suggests age spreads much larger than this, and more comparable to a log(age) distribution with $\sigma=0.5$ (the dotted line in Fig. 18). We then conclude that unresolved binaries in our clusters cannot account for the observed age spread.

\section{Conclusions}

The main results of this work are the following:

- In the $J, H$, and $K_{\mathrm{s}}$ NIR bands, we have imaged 26 intermediate and high-mass star-forming regions selected from a larger sample of sources spanning a range in luminosities and presumed youth. We have identified the presence of 23 young stellar clusters in 22 fields.

- The detected clusters have richness indicator values of between ten and several tens of objects and have median radii of 0.7 pc. Compared to clusters around Herbig Ae/Be stars,

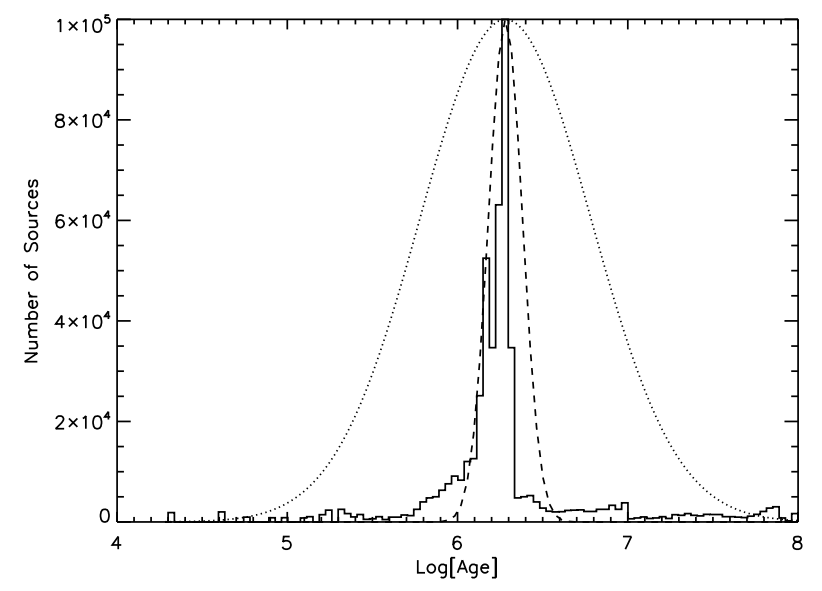

Fig. 18. Full line represents the age spread resulting from the simulations of Weidner et al. (2008) for a cluster with an input age of $2 \times 10^{6} \mathrm{yrs}$ and $100 \%$ binarity fraction. The two gaussians are the age weight functions used in our simulations of Gaussian Star Formation Histories, with $\sigma=0.1$ (dashed line) and 0.5 (dotted line) respectively. In case of constant SFH models, the adopted age spread is comparable to the $\sigma=0.5$ distribution in the figure.

our clusters seem richer and larger for any given mass for the most massive star in each cluster. Color-color diagrams show that these clusters are young: many sources exhibit colors typical of young pre-MS objects with an intrinsic IR excess originating in warm circumstellar dust. This is confirmed by the analysis of color-magnitude diagrams, where a significant fraction of stars in each cluster are found to be related to the Pre-MS evolutionary tracks, even after conservative de-reddening is applied.

- We have been unable to perform a direct inversion of stellar luminosities (and colors) into masses and ages; we use a synthetic cluster generator (SCG) model to create statisticallysignificant cluster simulations for different initial parameters (IMF, SFH, source ages, and their distribution), and compare the synthetic KLFs and HKCFs with the observed (field star-subtracted) ones. For the fraction of clusters for which this comparison selects a clearly defined region of the parameter space, we conclude that star formation in these regions cannot be represented by a single burst, but is a process that is spread out in time. Clusters have mean ages of a few $10^{6} \mathrm{yrs}$; the ages of most of the clusters members are spread, within each cluster, between a few $10^{5}$ yrs and a few $10^{6}$ yrs. Together with the independent evidence that the most massive stars in these systems are very young, or not even yet on the ZAMS, this result is difficult to reconcile with any model predicting cluster formation in a crossing time.

- The cluster radii seem to be inversely proportional to their age, as also confirmed by the comparison of cluster parameters with those typical of Ae/Be systems, which are smaller and less rich. As suggested by numerical simulations in the literature, dispersal of intra-cluster gas (by, e.g., molecular outflows or radiation fields from massive stars) may lead to the loss of a fraction of cluster stellar population, thus indeed leading to smaller and less rich clusters. Our results seem in line with this prediction.

- The relation between the mass of the most massive star in a cluster and the cluster's richness indicator suggests that a physical rather than statistical nature of the cluster origin is more likely. 
Acknowledgements. We thank the anonymous referee whose comments greatly helped improving the quality of the data presentation and the overall readability of the paper. We also thank P. Kroupa for his comments and suggestions.

\section{References}

Baumgardt, H., \& Kroupa, P. 2007, MNRAS, 380, 1589

Behrend, R., \& Meader, A. 2001, A\&A, 373, 190

Beltrán, M. T., Cesaroni, R., Neri, R., et al. 2004, ApJ, 601, L187

Beltrán, M. T., Brand, J., Cesaroni, R., et al. 2006, A\&A, 447, 221

Benjamin, R. A., Churchwell, E. B., Babler, B., et al. 2003, PASP, 115, 953

Bertoldi, F., \& McKee, C. F. 1992, ApJ 395, 140

Bessel, M. S. 1979, PASP, 91, 377

Bessel, M. S. 1991, AJ, 102, 303

Bessel, M. S., \& Brett, J. M. 1988, PASP, 100, 1134

Beuther, H., Schilke, P., Sridharan, T. K., et al. 2002, A\&A, 383, 892

Binney, S. J., \& Tremaine, S. 1987, Galactic Dynamics (Princeton NJ: Princeton Univ. Press)

Bonnell, I. A., \& Bate, M. R. 2002, MNRAS, 336, 659

Bonnell, I. A., \& Bate, M. R. 2006, MNRAS, 370, 488

Bonnell, I. A., \& Clarke, C. 1999, MNRAS, 309, 461

Bonnell, I. A., \& Davies, M. B. 1998, MNRAS, 295, 691

Bonnell, I. A., Bate, M. R., \& Zinnecher, H. 1998, MNRAS, 298, 93

Bonnell, I. A., Bate, M. R., Clarke, C., \& Pringle, J. E. 2001, MNRAS, 323, 785

Bonnell, I. A., Bate, M. R., \& Vine, S. G. 2003, MNRAS, 343, 413

Bonnell, I. A., Vine, S. G., \& Bate, M. R. 2004, MNRAS, 349, 735

Brand, J., \& Blitz, L. 1993, A\&A, 275, 67

Brand, J., Cesaroni, R., Palla, F., \& Molinari, S. 2001, A\&A, 370, 230

Cesaroni, R. 2005, Ap\&SS, 295, 5

Cesaroni, R., Neri, R., Olmi, L., et al. 2005, A\&A, 434, 1039

Cesaroni, R., Galli, D., Lodato, G., Walmsley, C. M., \& Zhang, Q. 2006, Protostars \& Planet V, Disks Around Young O-B (Proto)Stars: Observation and Theory

Chini, R., Hoffmeister, V., Kimeswenger, S., et al. 2004, Nature, 429, 155

Clarke, C., \& Delgrado-Donate, E. 2003, Galactic Star Formation Across the Stellar Mass Spectrum, ASP Conf. Ser., 287

de Wit, W.-J., Testi, L., Palla, F., \& Zinnecker, H. 2005, A\&A, 437, 247

Hartmann, L. W., Jones, B. F., Stauffer, J. R., \& Kenion, S. J. 1991, AJ, 101, 1050

Hillenbrand, L. A., \& Carpenter, J. M. 2000, ApJ, 540, 236

Hoogerwerf, R., de Bruijne, J. H. J., \& de Zeeuw, P. T. 2001, A\&A, 365, 49

Hillenbrand, L. A. 1997, AJ, 113, 1733

Hunt, L. K., Mannucci, F., Testi, L., et al. 1998, AJ, 115, 259

Isobe, T., Feigelson, E. D., Akritas, M. G., \& Babu, G. J. 1990, ApJ, 364, 104

Klein, R. I., Inutsuka, S., Padoan, P., \& Tomisaka, K. 2006, Protostars \& Planet $\mathrm{V}$, Current Advances in the Methodology and Computational Simulation of the Formation of Low-Mass Stars
Kenyon, S., \& Hartmann, L. 1995, ApJS, 101, 117

Keto, E. 2003, ApJ, 599, 1196

Koornneef, J. 1983, A\&A, 128, 84

Kroupa, P., Tout, C. A., \& Gilmore, G. 1993, MNRAS, 262, 545

Kumar, M. S. N., Keto, E., \& Clerkin, E. 2006, A\&A, 449, 1033

Kumar, M. S. N., \& Grave, J. M. C. 2008, ASPC, 387, 323

Lada, C., \& Adams, F. 1992, ApJ, 393, 278

Lada, C. J., \& Lada, E. A. 2003, ARA\&A, 41, 57

McKee, C. F., \& Tan, J. C. 2003, ApJ, 585, 850

Molinari, S., Brand, J., Cesaroni, R., \& Palla, F. 1996, A\&A, 308, 573

Molinari, S., Brand, J., Cesaroni, R., Palla, F., \& Palumbo, G. G. C. 1998, A\&A, 336, 339

Molinari, S., Brand, J., Cesaroni, R., \& Palla, F. 2000, A\&A, 355, 617

Molinari, S., Testi, L., Rodrguez, L. F., \& Zhang, Q. 2002, ApJ, 570, 758

Molinari, S., Pezzuto, S., Cesaroni, R., et al. 2008a, A\&A, 481, 345

Molinari, S., Faustini, F., Testi, L., et al. 2008b, A\&A, 487, 1119

Moraux, E., Lawson, W. A., \& Clarke, C. 2007, A\&A, 473, 163

Nakano, T. 1989, ApJ, 345, 464

Palla, F., \& Stahler, S. W. 1999, ApJ, 525, 722

Preibisch, Th., Ossenkopf, V., Yorke, H. W., \& Henning, Th. 1993, A\&A, 279, 577

Rieke, G. H., \& Lebofsky, M. J. 1985, ApJ, 288, 618

Salpeter, E. E. 1955, ApJ, 121, 161

Scalo, J. 1998, The Stellar Initial Mass Function, ed. G. Gilmore, \& D. Howell, 142,201

Strom, K. M., Strom, S. E., Edwards, S., Cabrit, S., \& Skrutskie, M. F. 1989, AJ, 97, 111

Tan, J. C. 2005, Cores to Clusters: Star Formation with Next Generation Telescopes, Astrophys. Space Sci. Lib., 324, 87

Tan, J. C., \& McKee, C. F. 2002, Hot Star Workshop III: The earliest Stages of Massive Star Birth, ASP Conf. Proc., 267, 267

Tan, J. C., Krumholz, M. R., \& McKee, C. F. 2006, ApJ

Testi, L., Palla, F., \& Natta, A. 1998, A\&AS, 133, 81

Testi, L., Palla, F., \& Natta, A. 1999, A\&A, 342, 515

Testi, L., Palla, F., \& Natta, A. 2001, From Darkness to Light, ed. T. Montmerle, \& Ph. André, ASP Conf. Ser., 243, 377

Testi, L., Palla, F., Prusti, T., Natta, A., \& Maltagliati, S. 1997, A\&A, 320, 159

Vig, S., Testi, L., Walmsley, M., et al. 2007, A\&A, 470, 977

Weidner, C., \& Kroupa, P. 2004, MNRAS, 348, 187

Weidner, C., \& Kroupa, P. 2006, MNRAS, 365, 1333

Weidner, C., Kroupa, P., \& Maschberger, T. 2008 [arXiv: 0811.3730W]

Yorke, H. W. 2002, Hot Star Workshop III: The earliest Stages of Massive Star Birth, ASP Conf. Proc., 267, 165

Zhang, Q., Hunter, T. R., Brand, J., et al. 2001, ApJ, 552, L167

Zhang, Q., Hunter, T. R., Brand, J., et al. 2005, ApJ, 625, 864

Zinnecker, H., \& Yorke, H. W. 2007, ARA\&A, 45, 481 
F. Faustini et al.: Properties of stellar clusters around high-mass young stars, Online Material p 1

\section{Appendix A: $K_{s}$ images for all observed fields}

This appendix contains the $K_{\mathrm{s}}$ images for all observed fields; these are also available at http://galatea.ifsi-roma. inaf.it/faustini/K-images/ 
F. Faustini et al.: Properties of stellar clusters around high-mass young stars, Online Material p 2

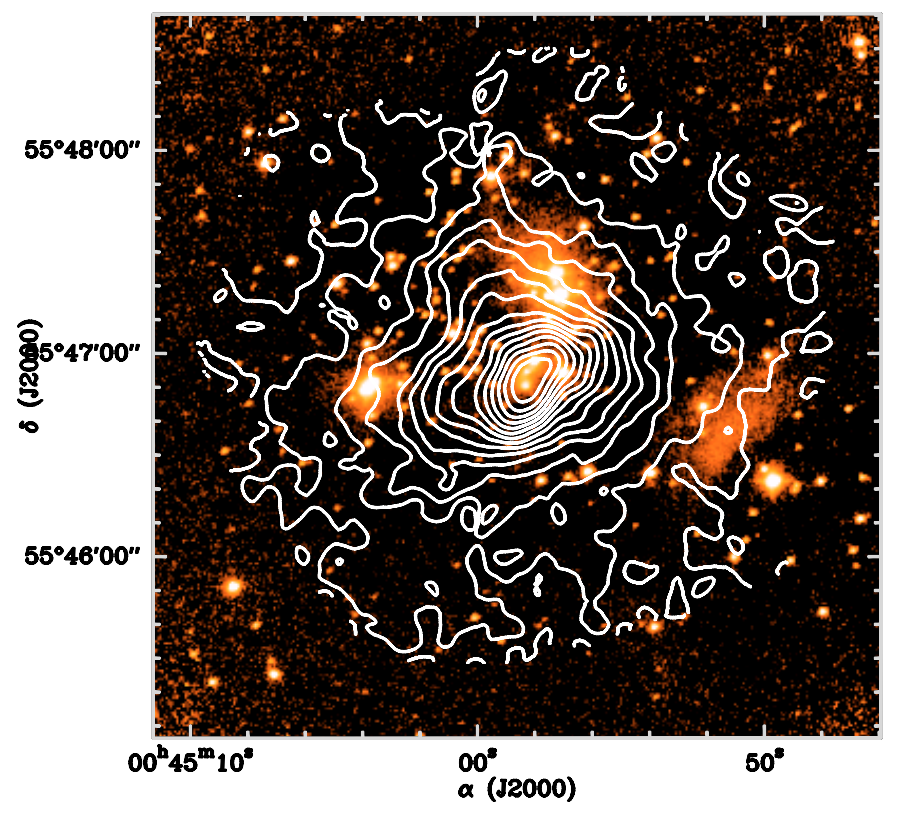

Fig. A.1. $K_{\mathrm{s}}$ image of field Mol003 with superimposed SCUBA $850 \mu \mathrm{m}$ continuum in white contours (Molinari et al. 2008a).

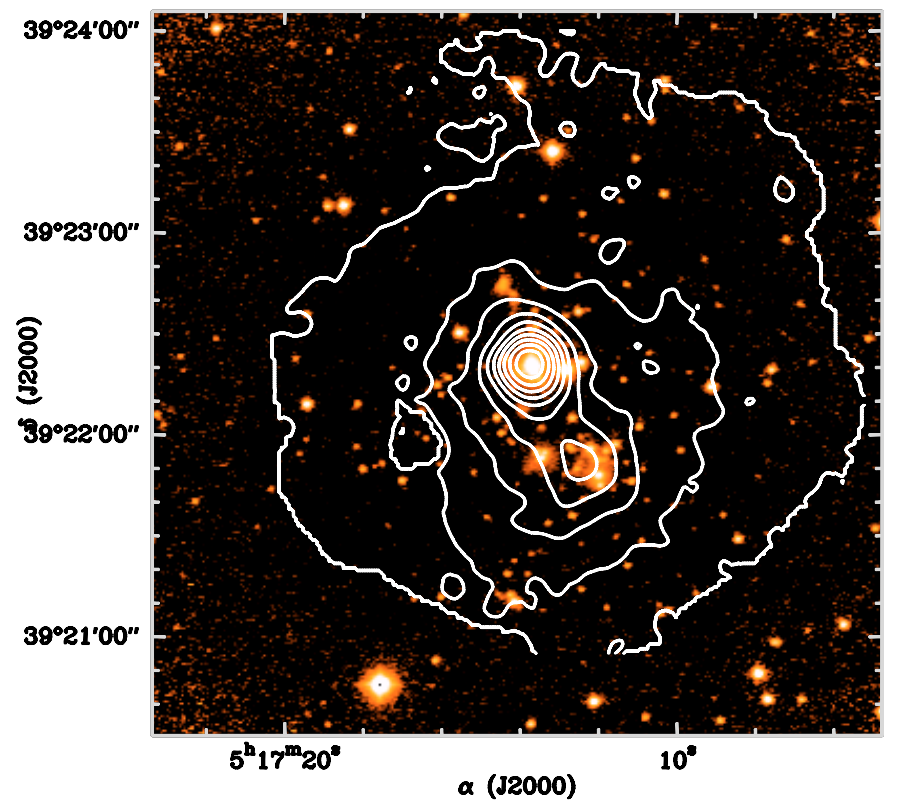

Fig. A.2. $K_{\mathrm{s}}$ image of field Mol008 with superimposed SCUBA $850 \mu \mathrm{m}$ continuum in white contours (Molinari et al. 2008a). 
F. Faustini et al.: Properties of stellar clusters around high-mass young stars, Online Material p 3

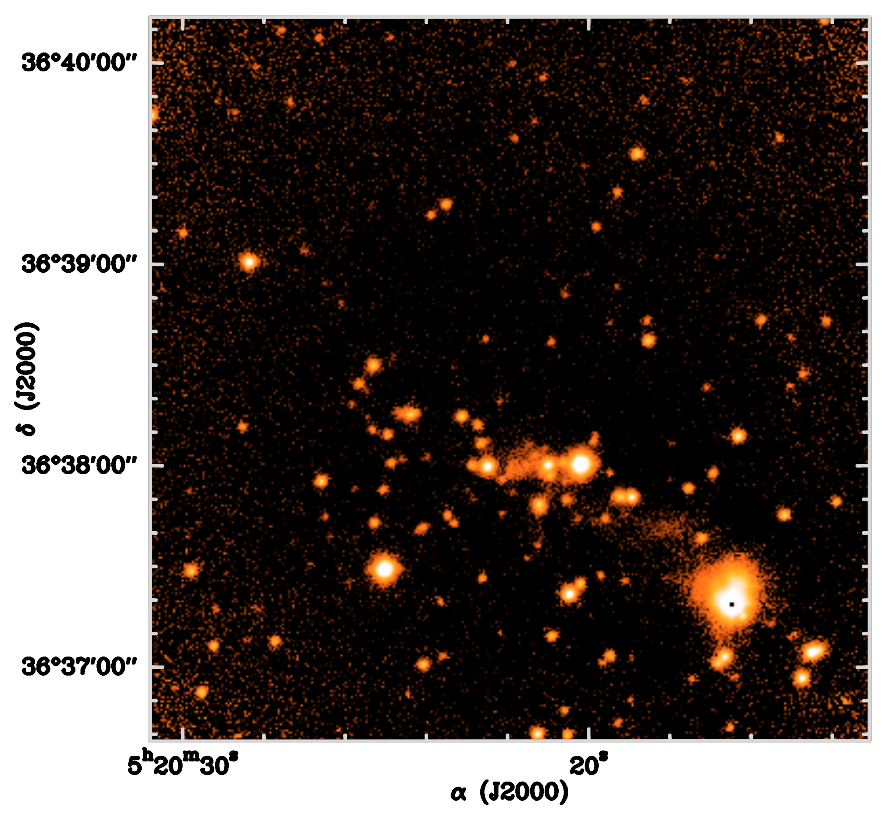

Fig. A.3. $K_{\mathrm{s}}$ image of field Mol009; no submillimeter image is available for this field.

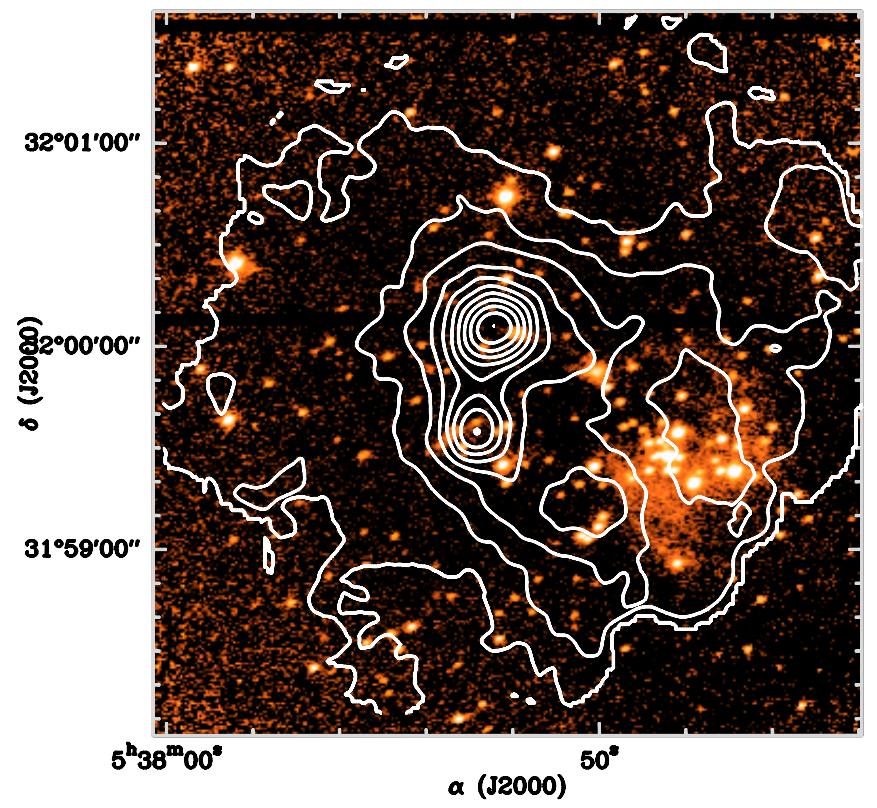

Fig. A.4. $K_{\mathrm{s}}$ image of field Mol011 with superimposed SCUBA $850 \mu \mathrm{m}$ continuum in white contours (Molinari et al. 2008a). 
F. Faustini et al.: Properties of stellar clusters around high-mass young stars, Online Material p 4

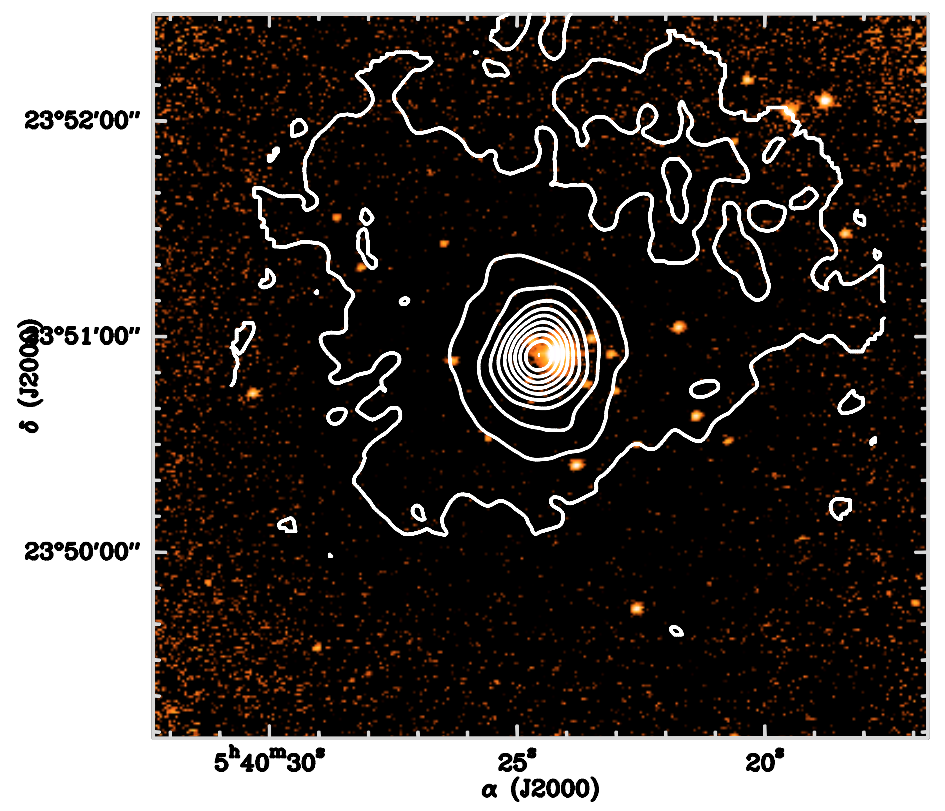

Fig. A.5. $K_{\mathrm{s}}$ image of field Mol012 with superimposed SCUBA $850 \mu \mathrm{m}$ continuum in white contours (Molinari et al. 2008a).

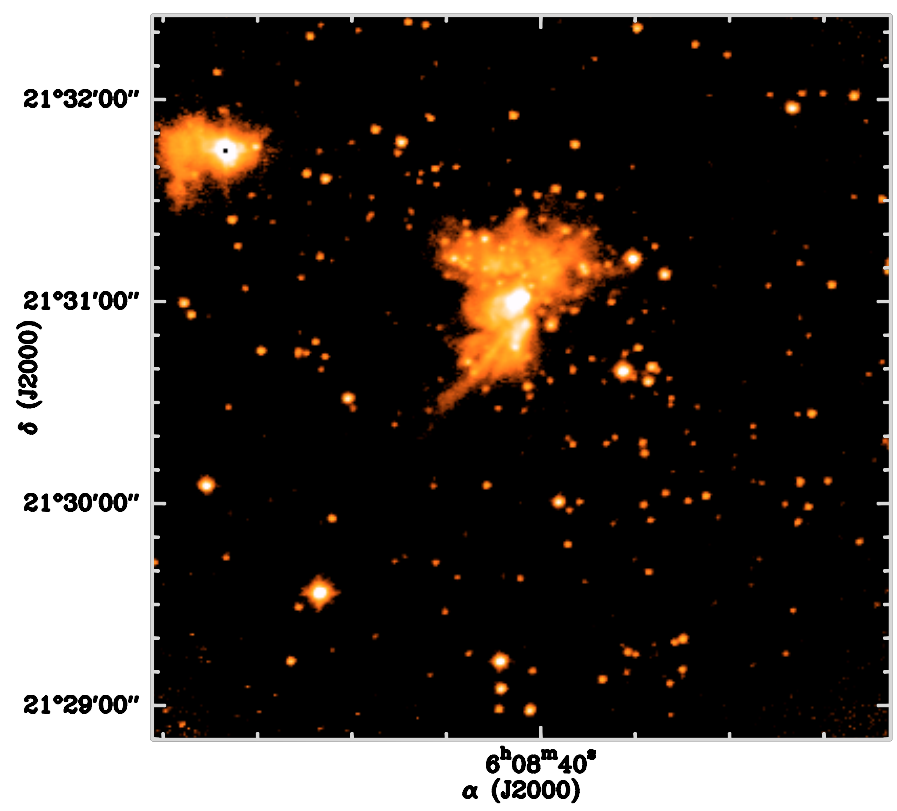

Fig. A.6. $K_{\mathrm{s}}$ image of field Mol015; no submillimeter image is available for this field. 
F. Faustini et al.: Properties of stellar clusters around high-mass young stars, Online Material p 5

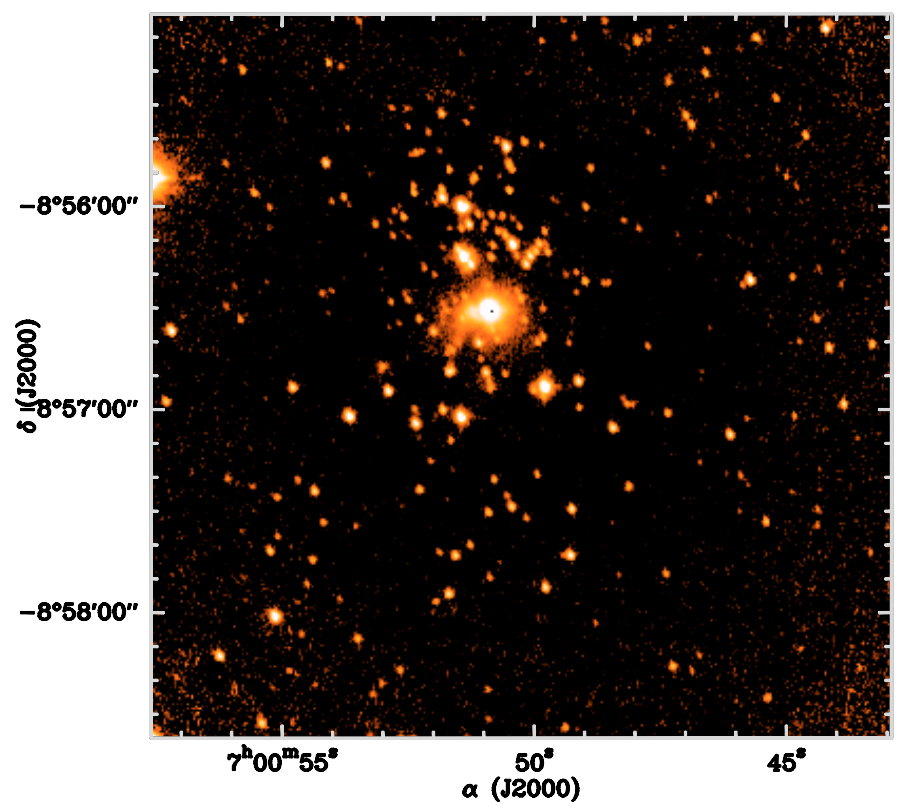

Fig. A.7. $K_{\mathrm{s}}$ image of field Mol028; no submillimeter image is available for this field.

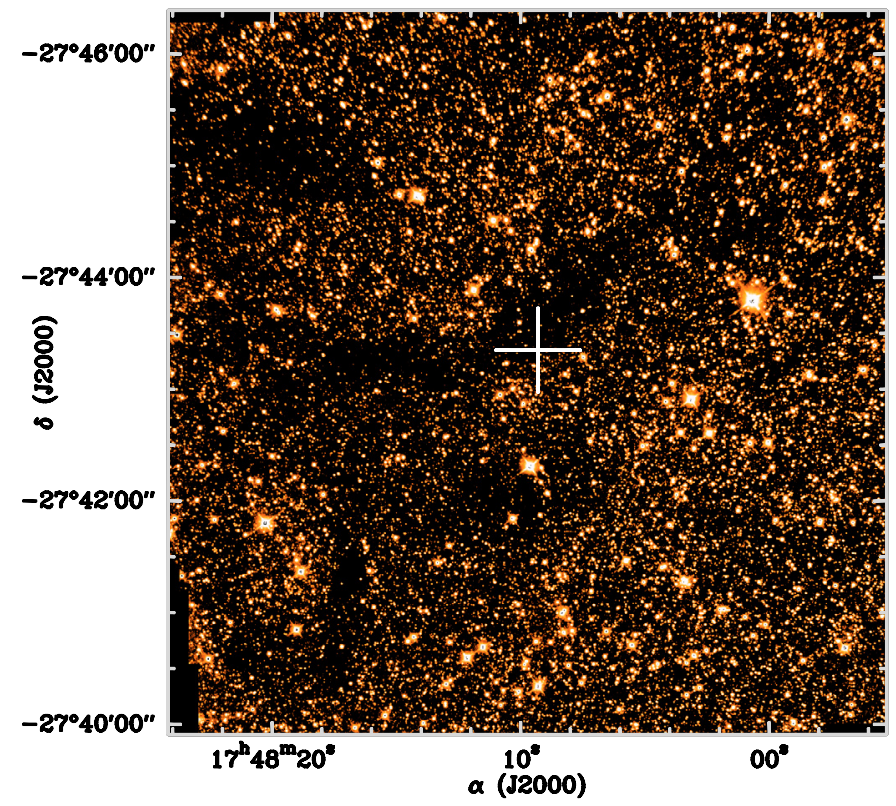

Fig. A.8. $K_{\mathrm{s}}$ image of field Mol030; no submillimeter image is available for this field. The white cross marks the position of the IRAS source. 


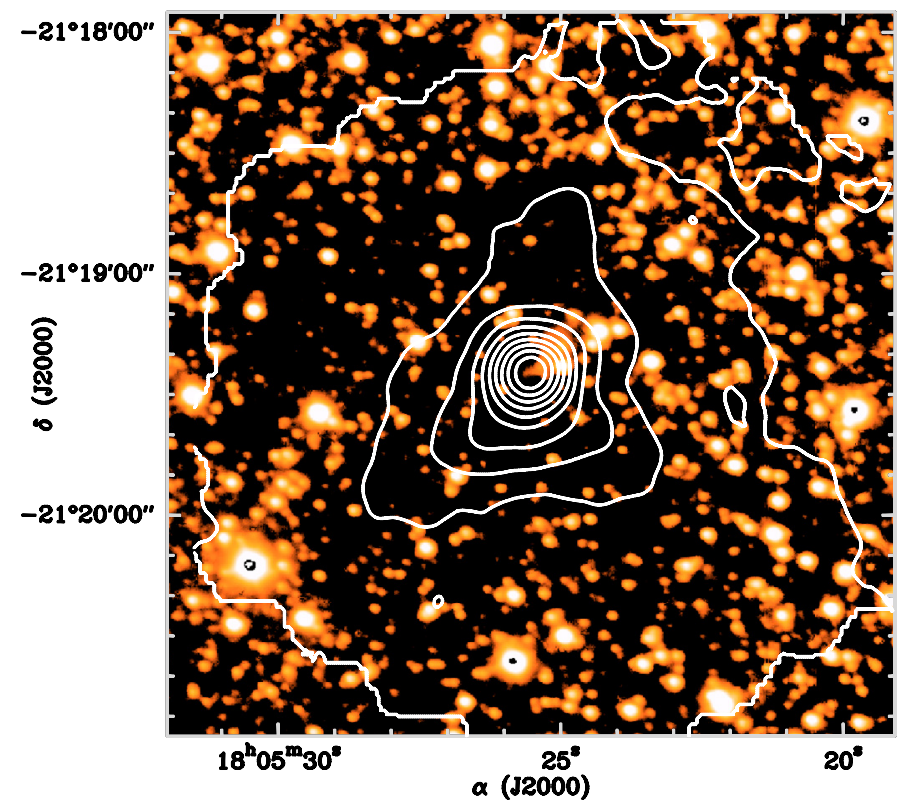

Fig. A.9. $K_{\mathrm{s}}$ image of field Mol038 with superimposed SCUBA $850 \mu \mathrm{m}$ continuum in white contours (Molinari et al. 2008a).

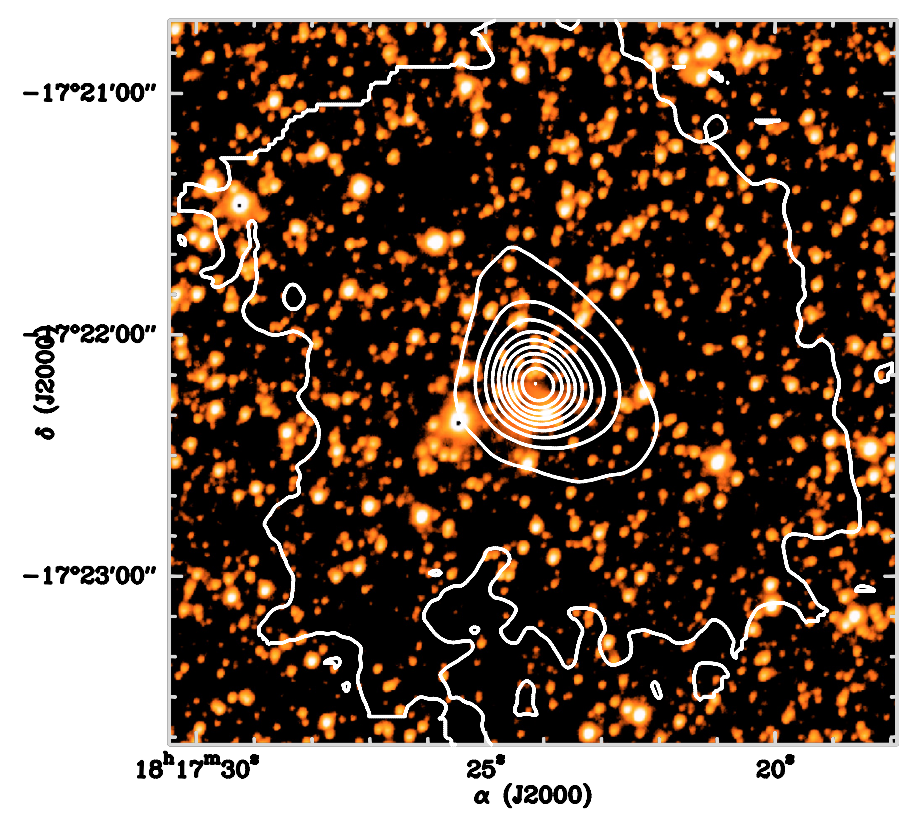

Fig. A.10. $K_{\mathrm{s}}$ image of field Mol045 with superimposed SCUBA $850 \mu \mathrm{m}$ continuum in white contours (Molinari et al. 2008a). 


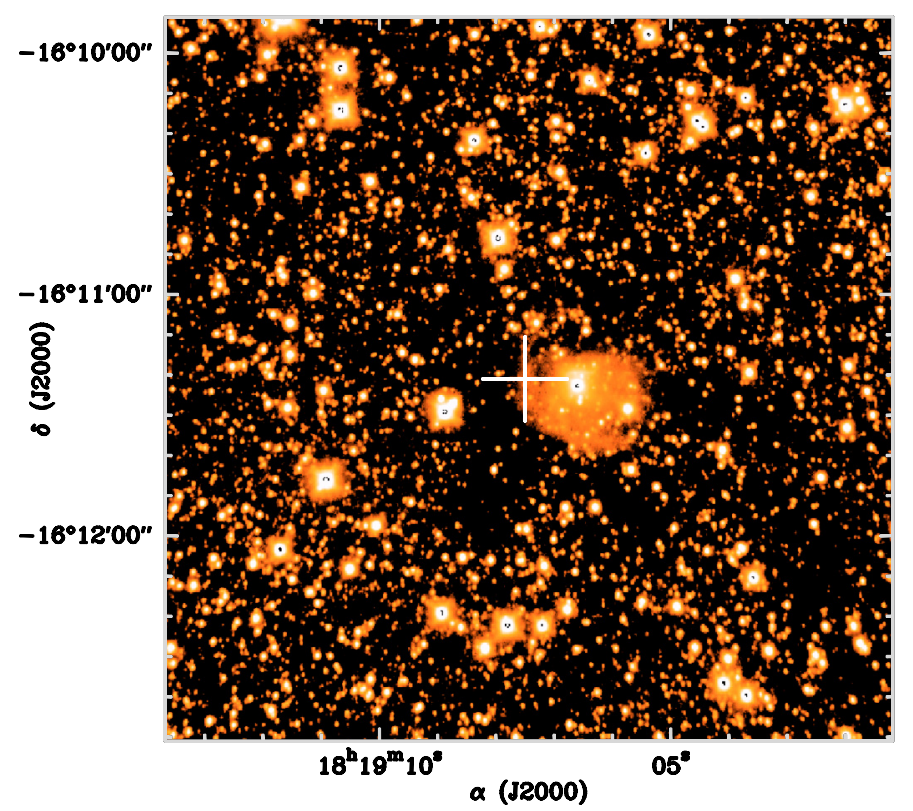

Fig. A.11. $K_{\mathrm{s}}$ image of field Mol050; no submillimeter image is available for this field. The white cross marks the position of the IRAS source.

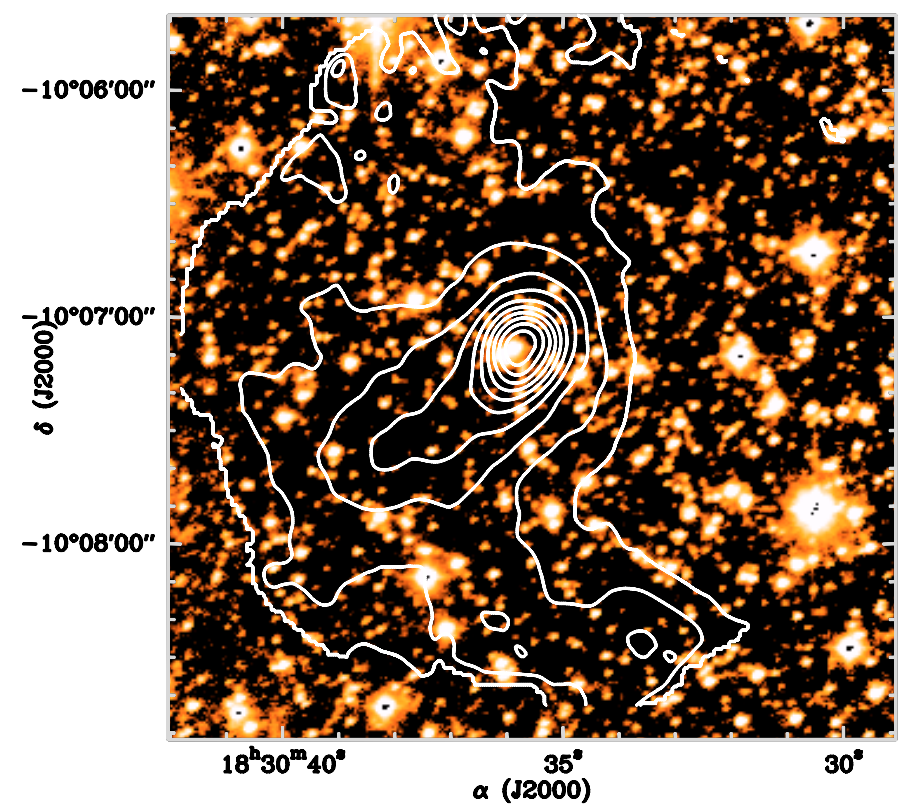

Fig. A.12. $K_{\mathrm{s}}$ image of field Mol059 with superimposed SCUBA $850 \mu \mathrm{m}$ continuum in white contours (Molinari et al. 2008a). 
F. Faustini et al.: Properties of stellar clusters around high-mass young stars, Online Material p 8

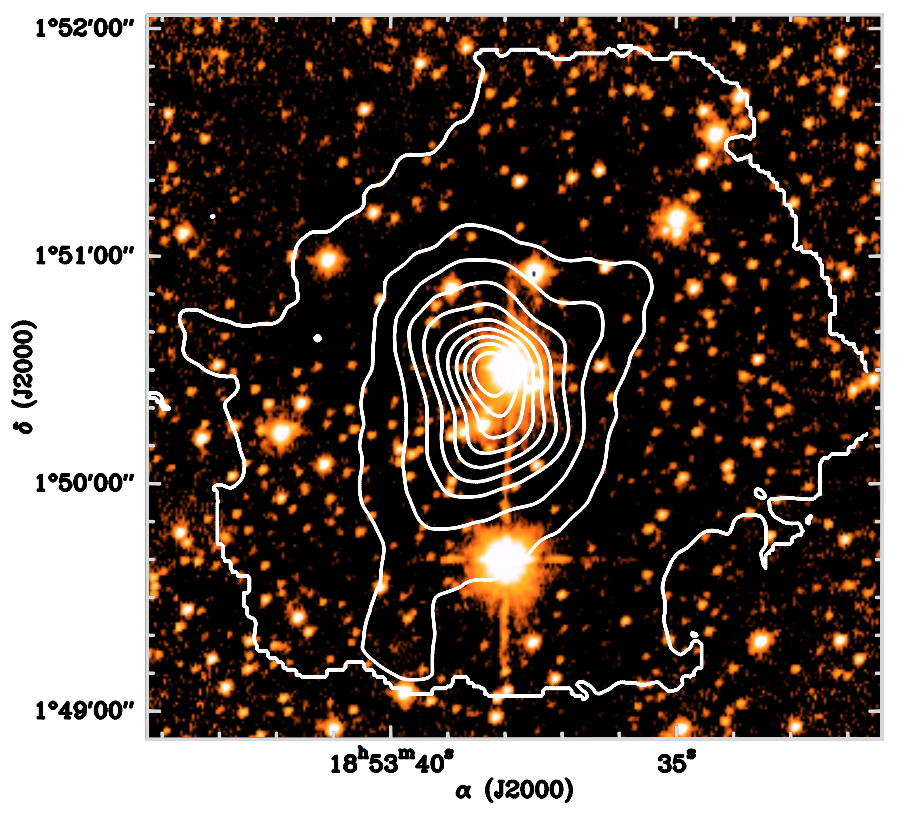

Fig. A.13. $K_{\mathrm{s}}$ image of field Mol075 with superimposed SCUBA $850 \mu \mathrm{m}$ continuum in white contours (Molinari et al. 2008a).

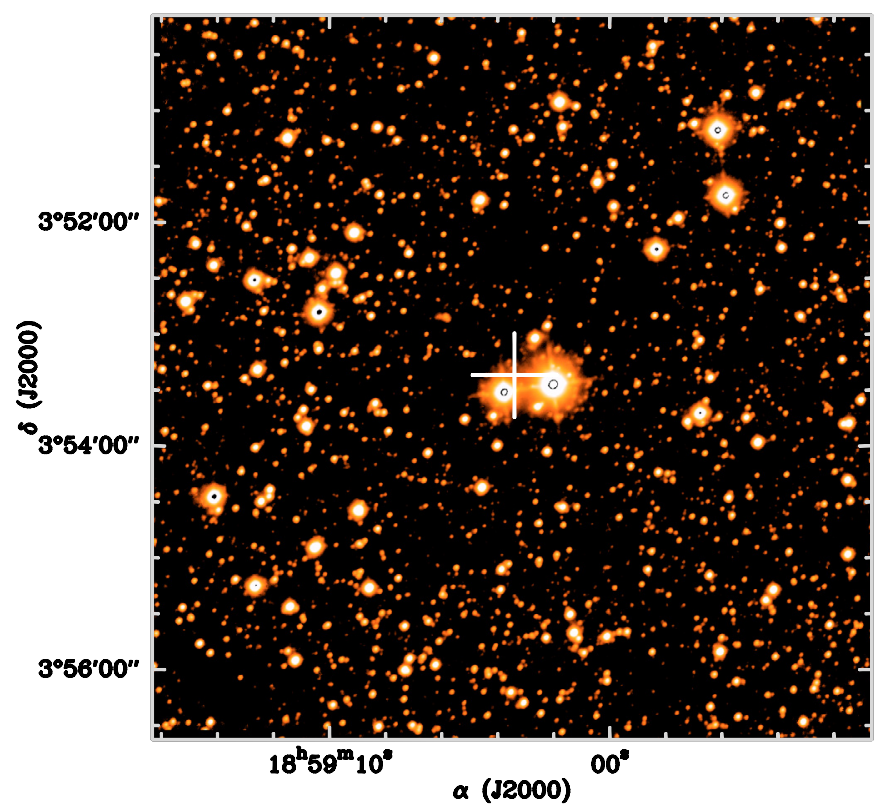

Fig. A.14. $K_{\mathrm{S}}$ image of field Mol082; no submillimeter image is available for this field. The white cross marks the position of the IRAS source. 
F. Faustini et al.: Properties of stellar clusters around high-mass young stars, Online Material p 9

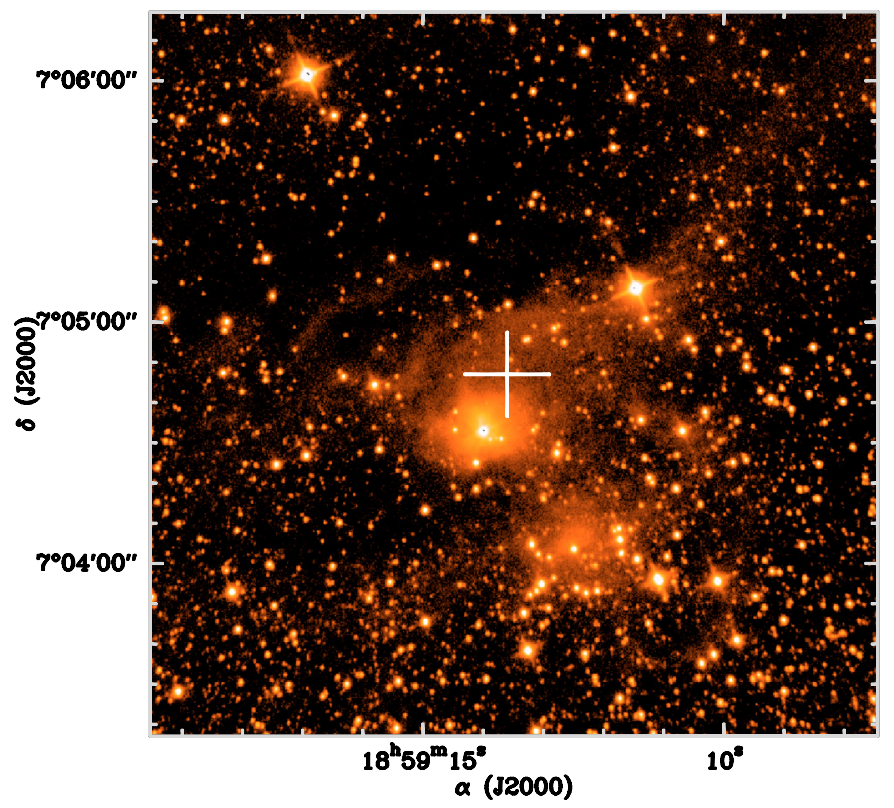

Fig. A.15. $K_{\mathrm{s}}$ image of field Mol084; no submillimeter image is available for this field. The white cross marks the position of the IRAS source.

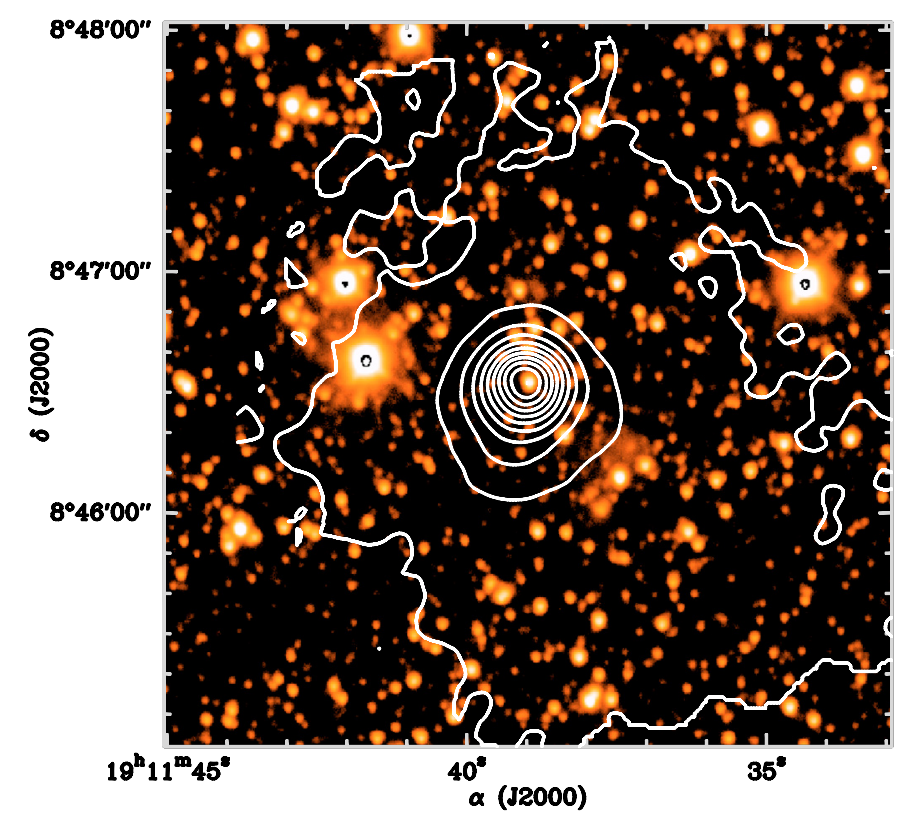

Fig. A.16. $K_{\mathrm{s}}$ image of field Mol098 with superimposed SCUBA $850 \mu \mathrm{m}$ continuum in white contours (Molinari et al. 2008a). 
F. Faustini et al.: Properties of stellar clusters around high-mass young stars, Online Material $p 10$

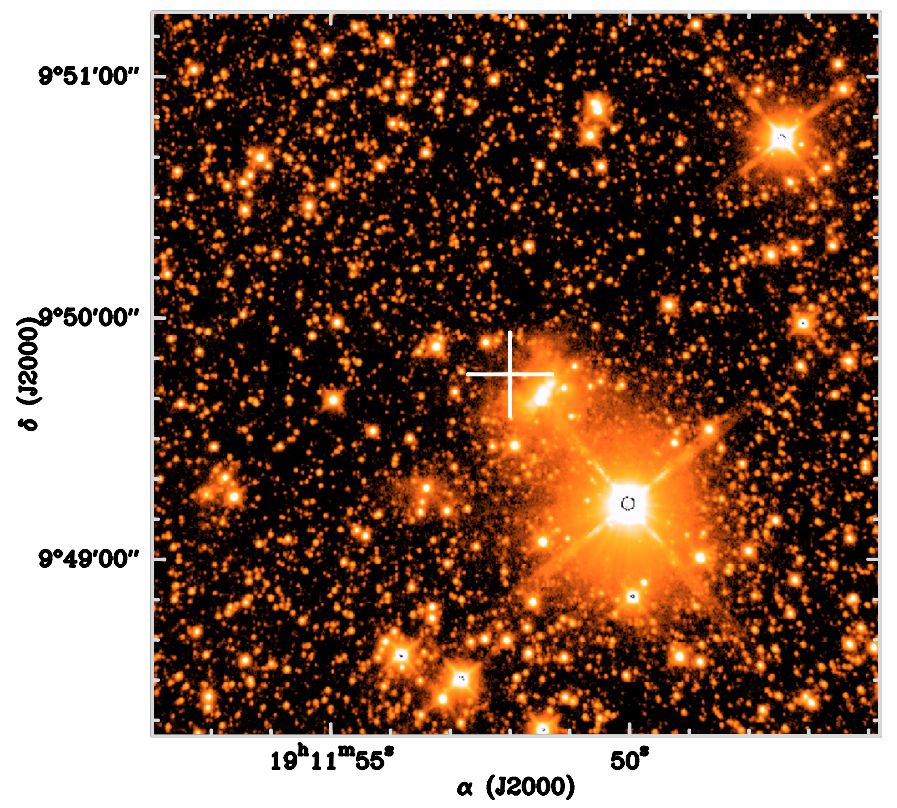

Fig. A.17. $K_{\mathrm{s}}$ image of field Mol099; no submillimeter image is available for this field. The white cross marks the position of the IRAS source.

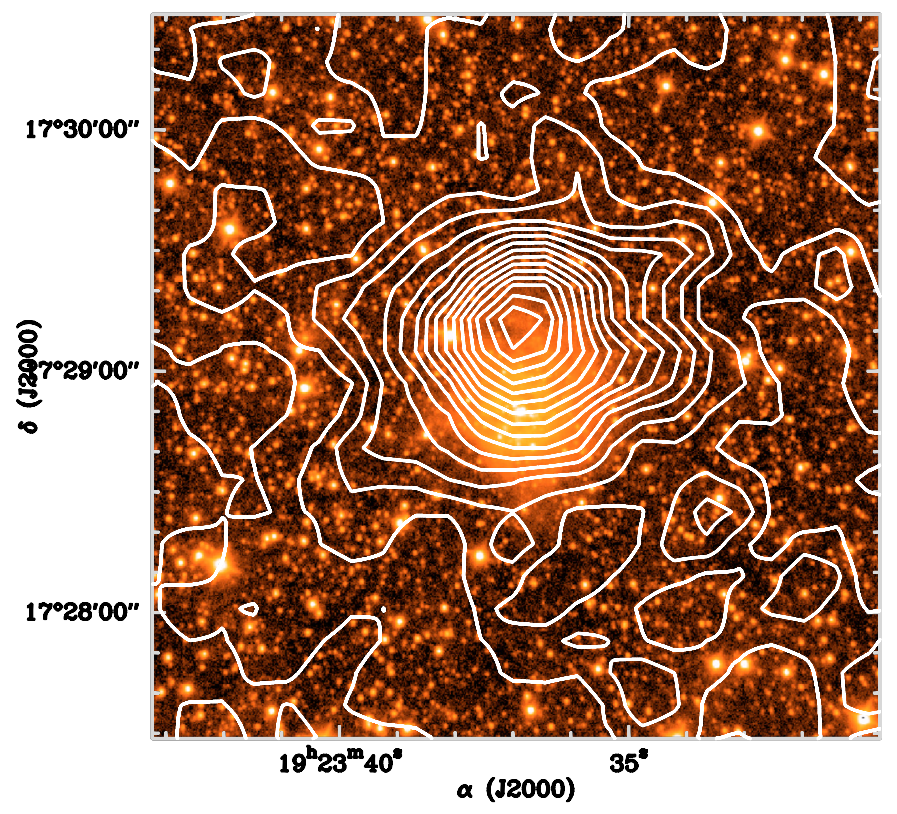

Fig. A.18. $K_{\mathrm{s}}$ image of field Mol103 with superimposed SIMBA 1.2mm continuum in white contours ( Beltrán et al. 2006). 
F. Faustini et al.: Properties of stellar clusters around high-mass young stars, Online Material p 11

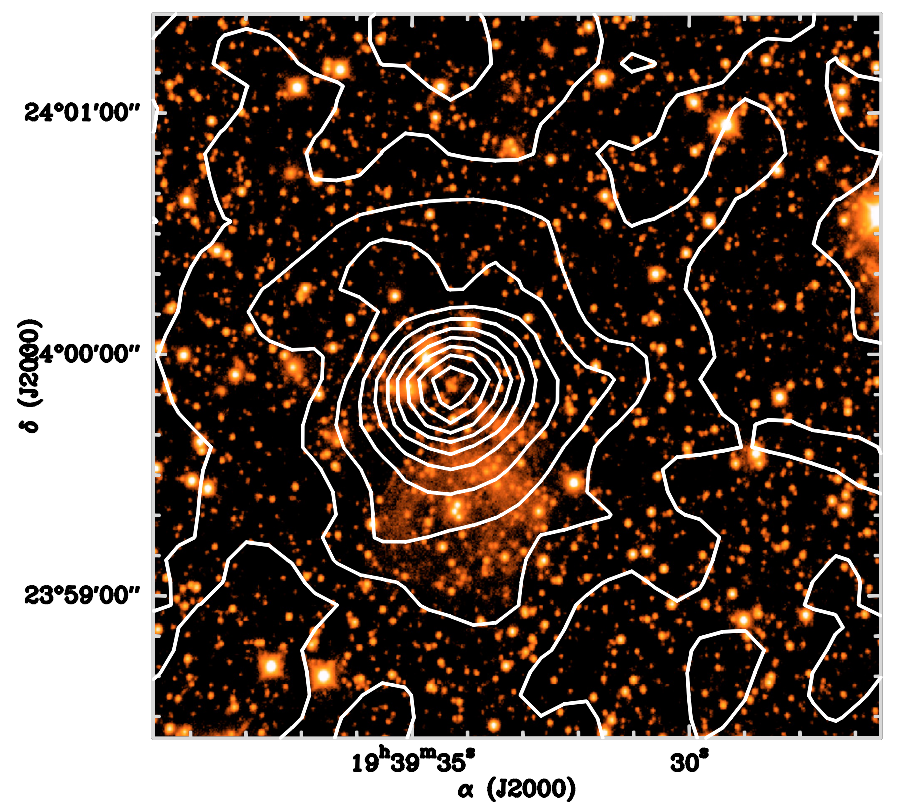

Fig. A.19. $K_{\mathrm{s}}$ image of field Mol109 with superimposed SIMBA 1.2mm continuum in white contours ( Beltrán et al. 2006).

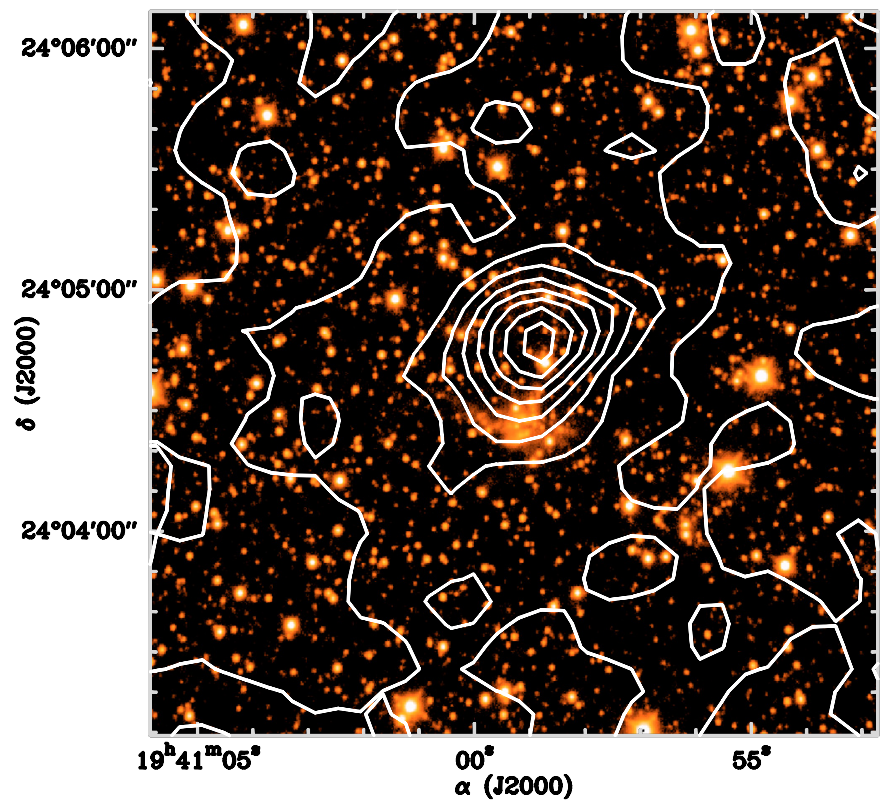

Fig. A.20. $K_{\mathrm{s}}$ image of field Mol110 with superimposed SIMBA $1.2 \mathrm{~mm}$ continuum in white contours ( Beltrán et al. 2006). 
F. Faustini et al.: Properties of stellar clusters around high-mass young stars, Online Material p 12

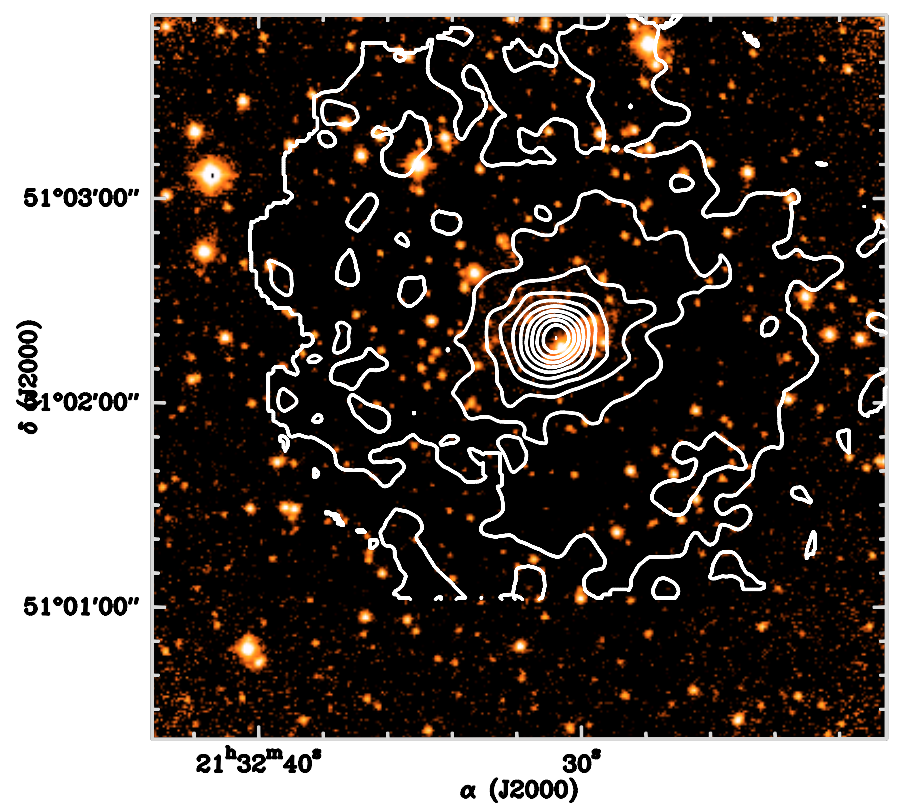

Fig. A.21. $K_{\mathrm{s}}$ image of field Mol136 with superimposed SCUBA $850 \mu \mathrm{m}$ continuum in white contours (Molinari et al. 2008a).

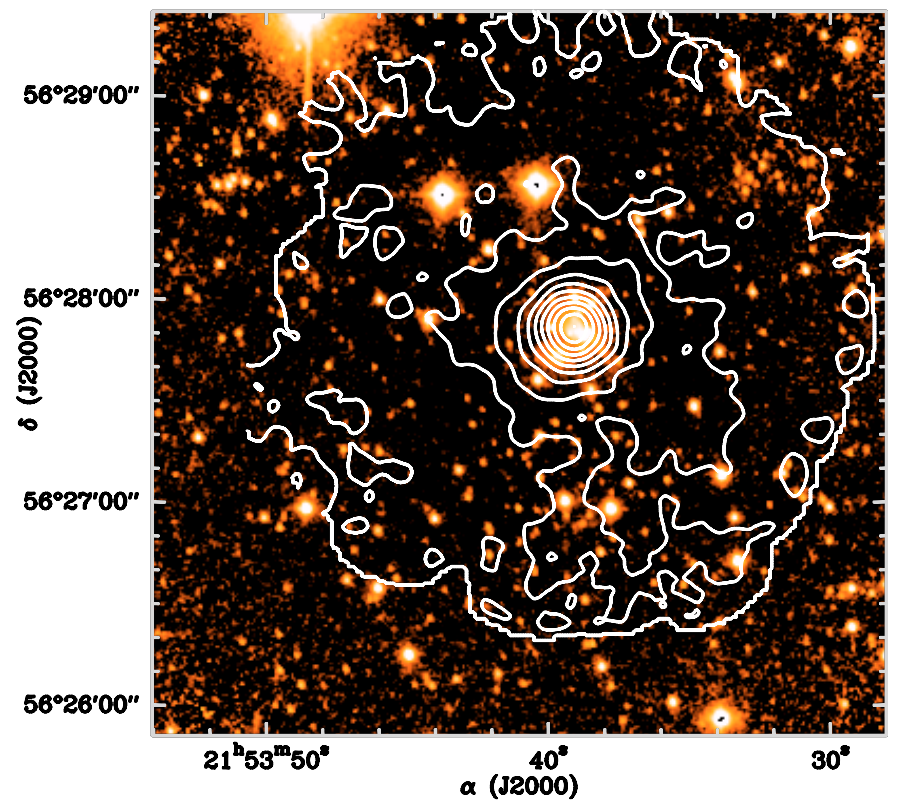

Fig. A.22. $K_{\mathrm{S}}$ image of field Mol139 with superimposed SCUBA $850 \mu \mathrm{m}$ continuum in white contours (Molinari et al. 2008a). 
F. Faustini et al.: Properties of stellar clusters around high-mass young stars, Online Material p 13

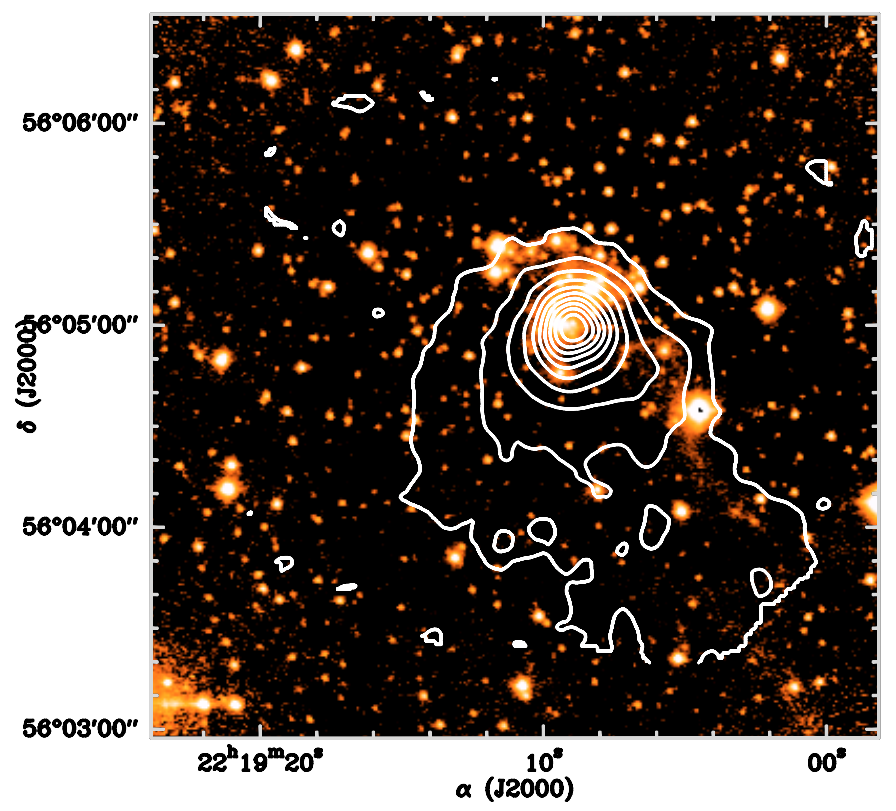

Fig. A.23. $K_{\mathrm{s}}$ image of field Mol143 with superimposed SCUBA $850 \mu \mathrm{m}$ continuum in white contours (Molinari et al. 2008a).

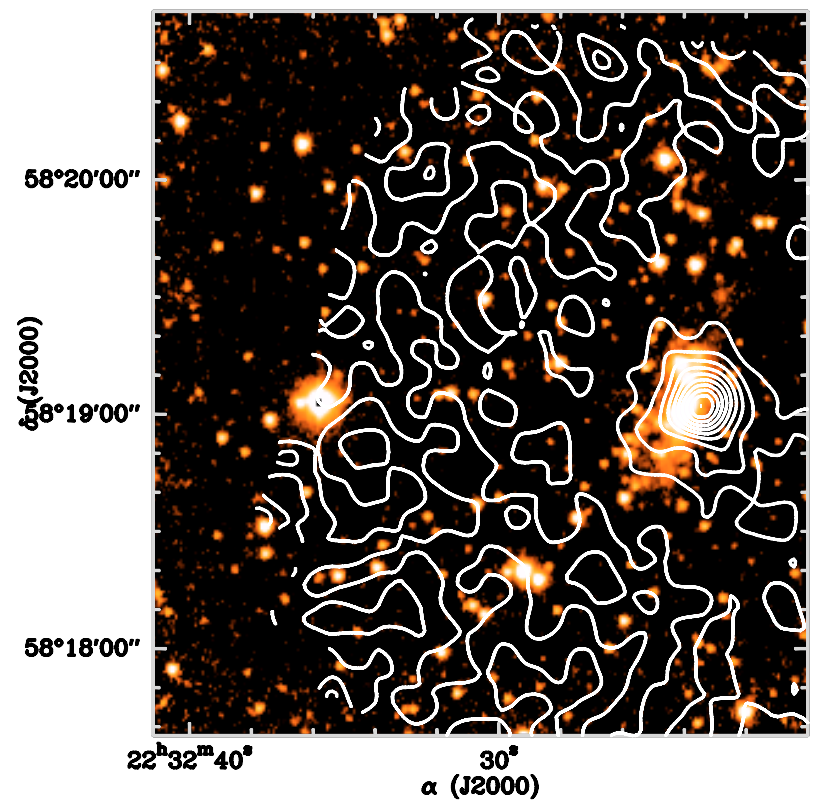

Fig. A.24. $K_{\mathrm{s}}$ image of field Mol148 with superimposed MAMBO $1.1 \mathrm{~mm}$ continuum in white contours (Molinari et al. 2008a). 
F. Faustini et al.: Properties of stellar clusters around high-mass young stars, Online Material p 14

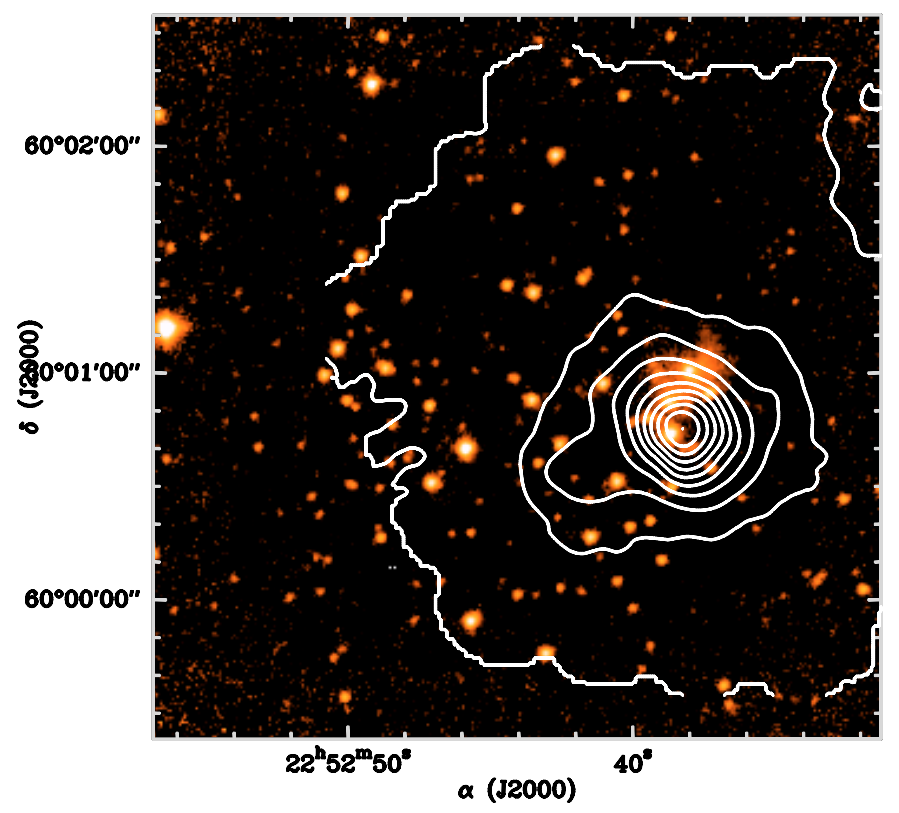

Fig. A.25. $K_{\mathrm{s}}$ image of field Mol151 with superimposed SCUBA $850 \mu \mathrm{m}$ continuum in white contours (Molinari et al. 2008a).

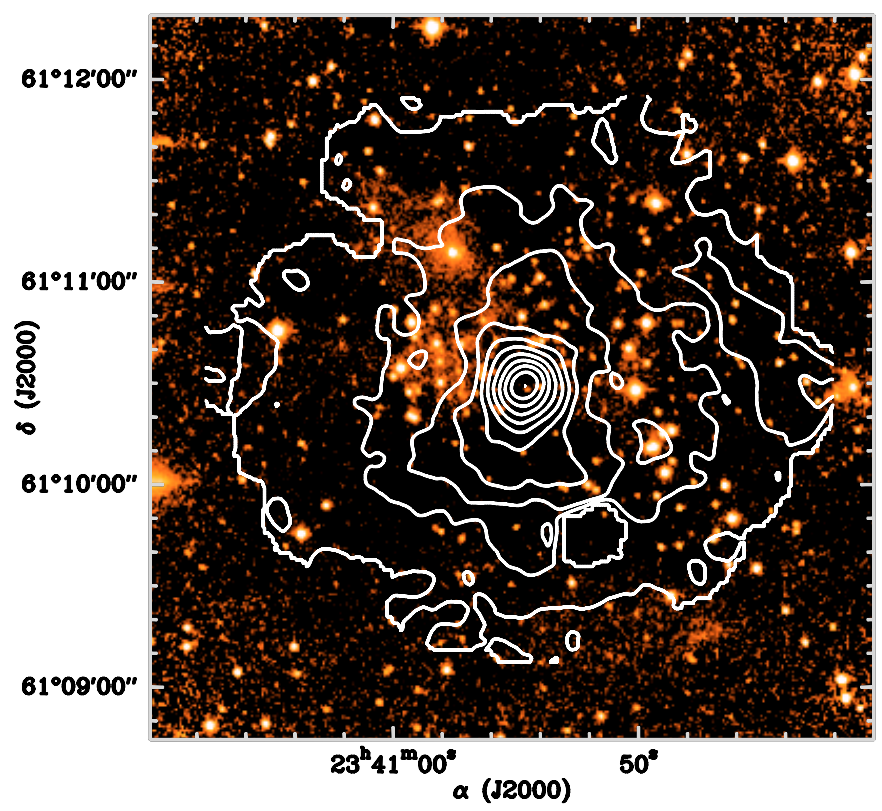

Fig. A.26. $K_{\mathrm{s}}$ image of field Mol160 with superimposed SCUBA $850 \mu \mathrm{m}$ continuum in white contours (Molinari et al. 2008a). 
F. Faustini et al.: Properties of stellar clusters around high-mass young stars, Online Material p 15

\section{Appendix $B: K_{s}$ luminosity functions}

This appendix presents the set of background-subtracted $K_{\mathrm{s}}$ luminosity functions for all detected clusters. Material can also be retrieved at http://galatea.ifsi-roma.inaf.it/ faustini/KLF/ 
F. Faustini et al.: Properties of stellar clusters around high-mass young stars, Online Material p 16
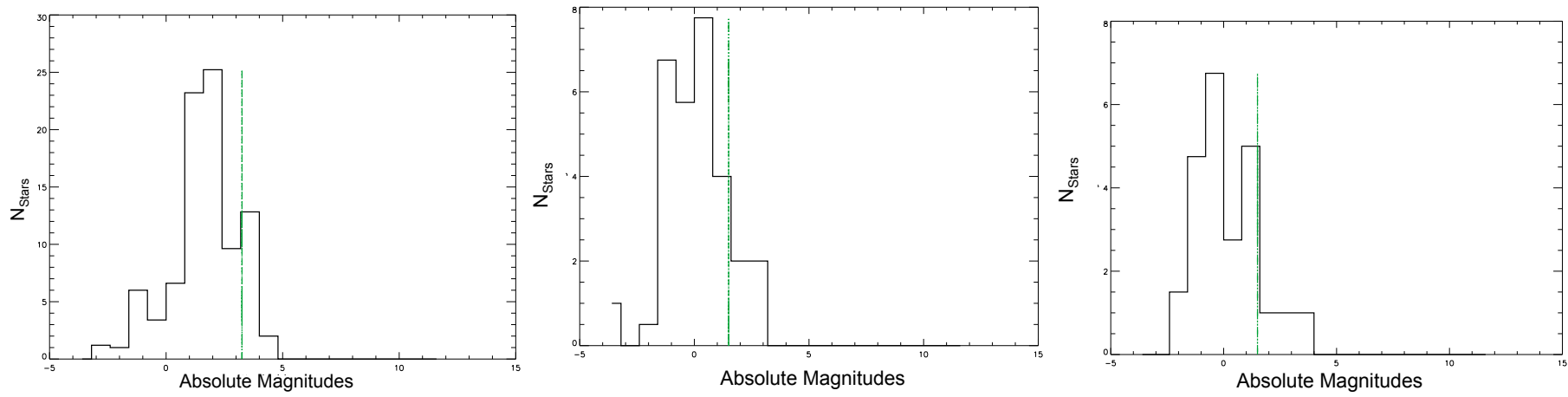

Fig. B.1. Mol003 (left), Mol008A (center), Mol008B (right)
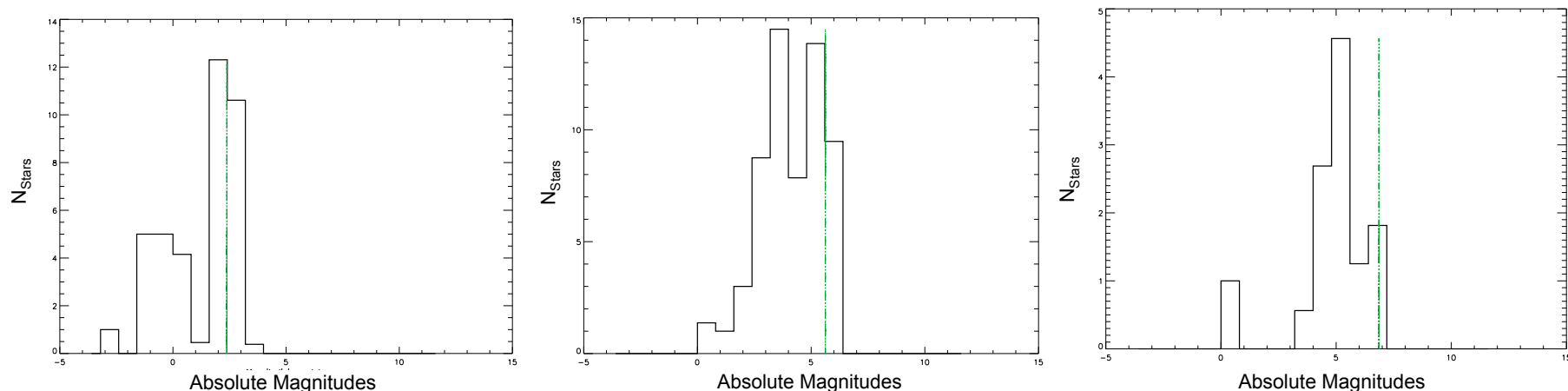

Fig. B.2. Mol009 (left), Mol011 (center), Mol012 (right).
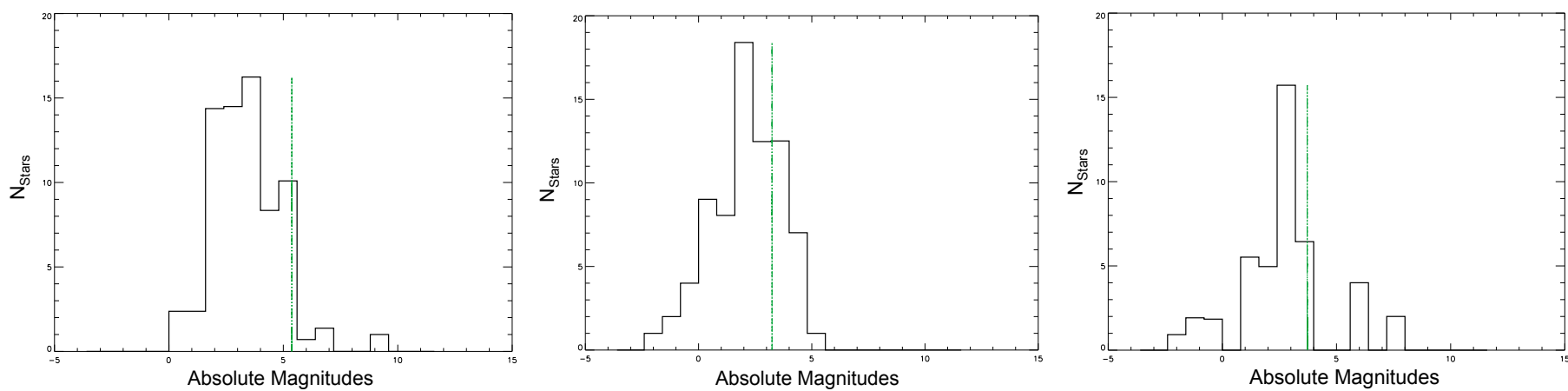

Fig. B.3. Mol015 (left), Mol028 (center), Mol045 (right).
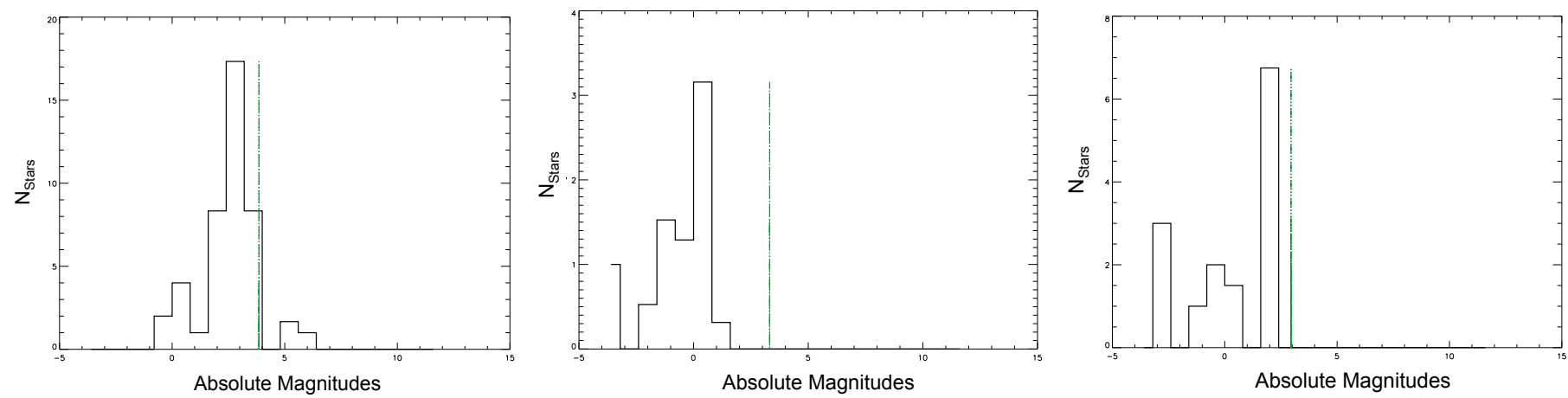

Fig. B.4. Mol050 (left), Mol075 (center), Mol082 (right). 
F. Faustini et al.: Properties of stellar clusters around high-mass young stars, Online Material p 17

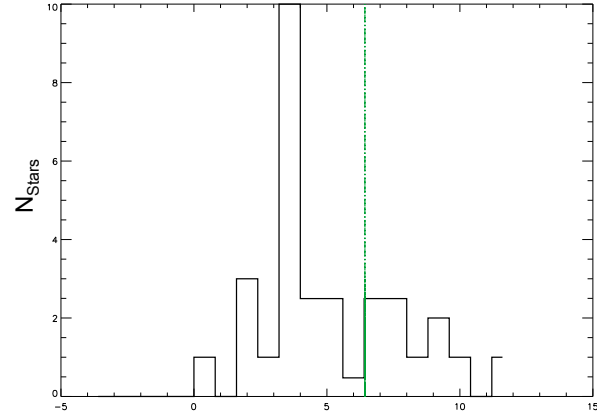

Absolute Magnitudes

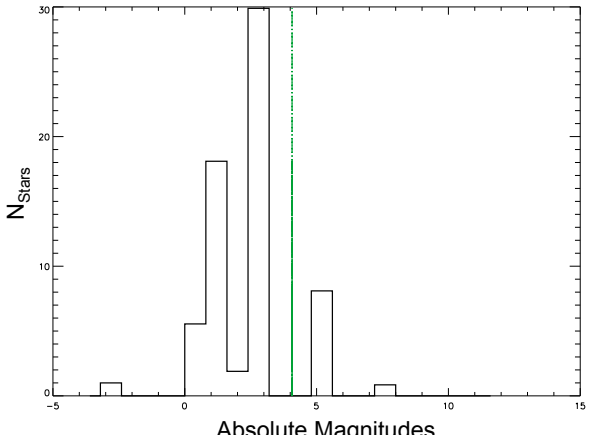

Absolute Magnitudes

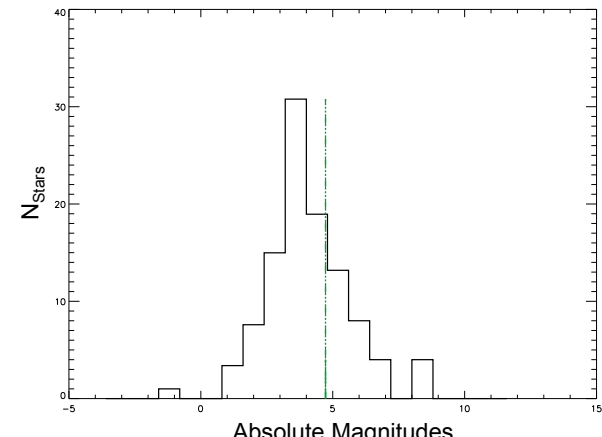

Absolute Magnitudes

Fig. B.5. Mol084 (left), Mol099 (center), Mol103 (right).

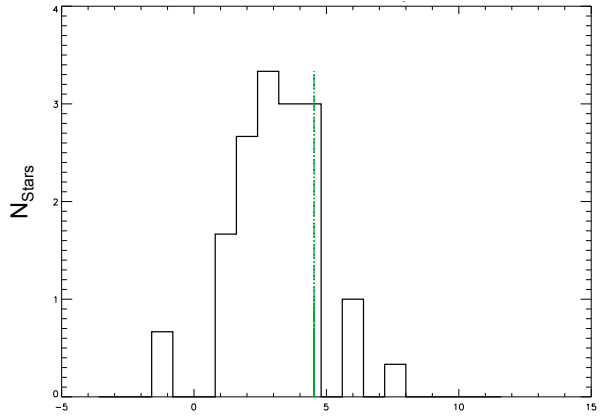

Absolute Magnitudes

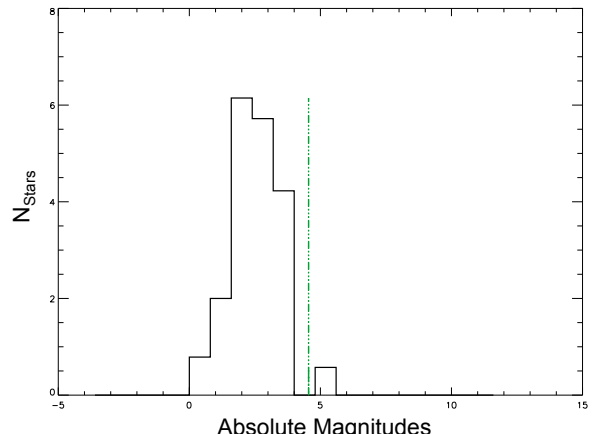

Absolute Magnitudes

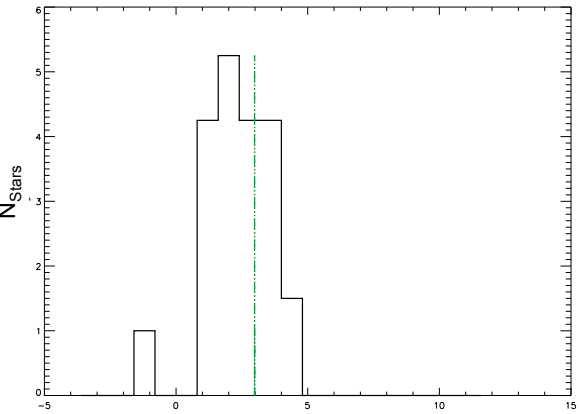

Absolute Magnitudes

Fig. B.6. Mol136 (left), Mol110 (center), Mol136 (right).

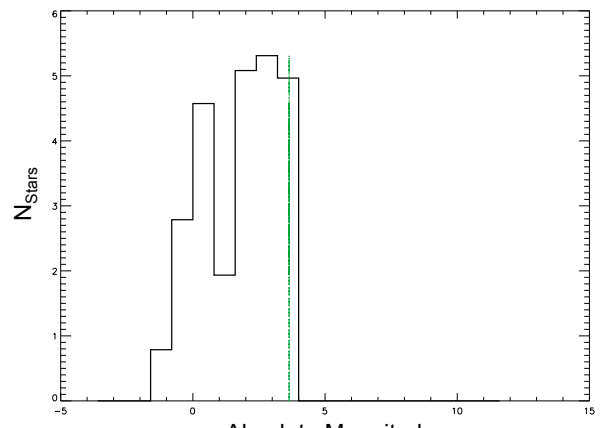

Absolute Magnitudes

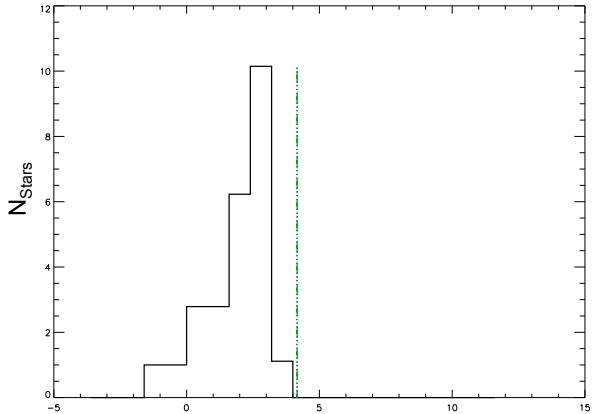

Absolute Magnitudes

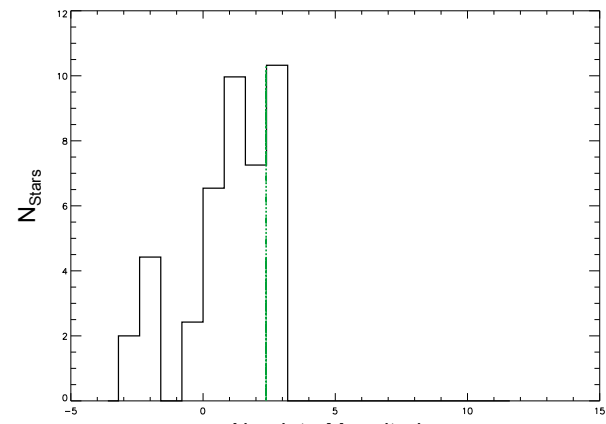

Absolute Magnitudes

Fig. B.7. Mol139 (left), Mol143 (center), Mol148 (right).

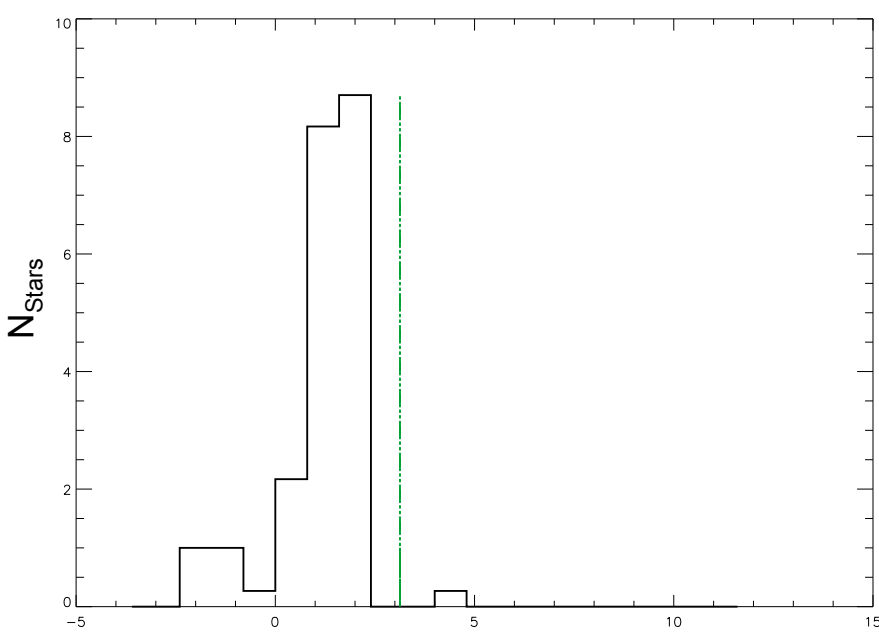

Absolute Magnitudes

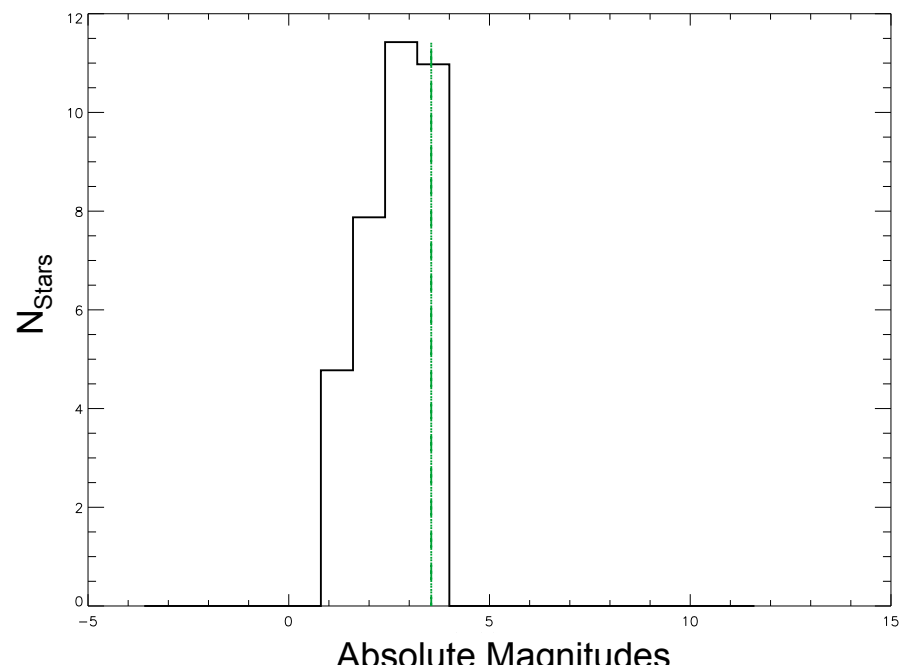

Absolute Magnitudes

Fig. B.8. Mol151 (left), Mol160 (right). 